\title{
Towards predictive data-driven simulations of wildfire spread - Part II: Ensemble Kalman Filter for the state estimation of a front-tracking simulator of wildfire spread
}

\author{
M. C. Rochoux ${ }^{1,2,3,4}$, C. Emery ${ }^{1,2,5}$, S. Ricci ${ }^{1,2}$, B. Cuenot $^{1}$, and A. Trouvé ${ }^{5}$ \\ ${ }^{1}$ CERFACS, 42 avenue Gaspard Coriolis, 31057 Toulouse, CEDEX 01, France \\ ${ }^{2}$ SUC/CNRS-URA1875, 42 avenue Gaspard Coriolis, 31057 Toulouse, CEDEX 01, France \\ ${ }^{3}$ Ecole Centrale Paris, Grande Voie des Vignes, 92295 Châtenay-Malabry, France \\ ${ }^{4}$ EM2C/CNRS-UPR288, Grande Voie des Vignes, 92295 Châtenay-Malabry, France \\ ${ }^{5}$ Dept. of Fire Protection Engineering, University of Maryland, College Park, Maryland, MD 20742, USA
}

Correspondence to: M. C. Rochoux (melanie.rochoux@graduates.centraliens.net)

Received: 27 April 2014 - Published in Nat. Hazards Earth Syst. Sci. Discuss.: 28 May 2014

Revised: 16 October 2014 - Accepted: 9 July 2015 - Published: 4 August 2015

\begin{abstract}
This paper is the second part in a series of two articles, which aims at presenting a data-driven modeling strategy for forecasting wildfire spread scenarios based on the assimilation of the observed fire front location and on the sequential correction of model parameters or model state. This model relies on an estimation of the local rate of fire spread (ROS) as a function of environmental conditions based on Rothermel's semi-empirical formulation, in order to propagate the fire front with an Eulerian front-tracking simulator. In Part I, a data assimilation (DA) system based on an ensemble Kalman filter (EnKF) was implemented to provide a spatially uniform correction of biomass fuel and wind parameters and thereby, produce an improved forecast of the wildfire behavior (addressing uncertainties in the input parameters of the ROS model only). In Part II, the objective of the EnKF algorithm is to sequentially update the twodimensional coordinates of the markers along the discretized fire front, in order to provide a spatially distributed correction of the fire front location and thereby, a more reliable initial condition for further model time-integration (addressing all sources of uncertainties in the ROS model). The resulting prototype data-driven wildfire spread simulator is first evaluated in a series of verification tests using synthetically generated observations; tests include representative cases with spatially varying biomass properties and temporally varying wind conditions. In order to properly account for uncertainties during the EnKF update step and to accurately represent
\end{abstract}

error correlations along the fireline, it is shown that members of the EnKF ensemble must be generated through variations in estimates of the fire's initial location as well as through variations in the parameters of the ROS model.

The performance of the prototype simulator based on state estimation (SE) or parameter estimation (PE) is then evaluated by comparison with data taken from a reduced-scale controlled grassland fire experiment. Results indicate that data-driven simulations are capable of correcting inaccurate predictions of the fire front location and of subsequently providing an optimized forecast of the wildfire behavior at future lead times. The complementary benefits of both PE and SE approaches, in terms of analysis and forecast performance, are also emphasized. In particular, it is found that the size of the assimilation window must be specified adequately with the persistence of the model initial condition and/or with the temporal and spatial variability of the environmental conditions in order to track sudden changes in wildfire behavior. The present prototype data-driven forecast system is still at an early stage of development. In this regard, this preliminary investigation provides valuable information on how to combine observations with a fire spread model in an efficient way, as well as guidelines to design the future system evolution in order to meet the operational requirements of wildfire spread monitoring. 


\section{Introduction}

Wildfires generally feature a front-like geometry and may be described at regional scales (i.e., at scales ranging from a few tens of meters up to several kilometers) as a thin flame zone that self-propagates normal to itself into unburnt vegetation. The local propagation speed is commonly referred to as the rate of spread (ROS) and is defined as the speed of the flame with respect to a fixed observer. Thus, the ROS can be regarded as the displacement rate of the flame surface separating the burning zone and the unburnt vegetation; it directly results from multi-scale multi-physics interactions between biomass pyrolysis, combustion and flow dynamics, radiation and convection heat transfer, as well as atmospheric dynamics and chemistry.

For early warning of potential wildfire danger, operational systems have been designed worldwide by national civil defense authorities to identify geographical areas that are subject to possibly extreme wildfire behavior. Fire danger is a generic term referring to the assessment of both constant and variable fire precursor factors affecting the ignition, spread, intensity and suppression capability of wildfires (Chandler et al., 1983). Current operational fire danger rating systems adopt a regional-scale viewpoint based on empirical ROS modeling approaches and integrate remote sensing data (i.e., meteorological, terrain topography and biomass fuel information) into a reduced set of macroscopic qualitative and/or numerical indices such as the Fire Weather Index (FWI) in Canada and in Europe (Van Wagner, 1987; Hirsch, 1996), the Forest Fire Danger Index (FFDI) in Australia (Noble et al., 1980; Dowdy et al., 2009) or the National Fire Danger Rating System (NFDRS) in the USA (Burgan, 1988). These fire danger rating systems mainly rely on meteorological information. Thus, the evaluation of fire danger could be improved through a more detailed wildfire spread modeling and a more accurate forecast of the potential ROS, accounting for the spatial and temporal variability of environmental conditions.

A wide range of wildfire spread modeling approaches has emerged over the last decade to describe the physical processes at flame scale as well as the interactions between the fire and the atmosphere. On the one hand, physics-based computational fluid dynamics (CFD) modeling approaches provide detailed numerical simulations of the combustionrelated processes that control the fire spread. Due to the high computational cost of flame-scale CFD and due to the lack of knowledge in environmental conditions, the use of CFDbased detailed modeling approaches such as FIRETEC (Linn et al., 2002), WFDS (Mell et al., 2007) or AVBP-PRISSMAPYROWO (Rochoux, 2014) is currently restricted to research projects and is not compatible with operational applications. On the other hand, regional-scale fire spread models such as FARSITE (Finney, 1998), FOREFIRE (Filippi et al., 2009, 2013), PROMETHEUS (Tymstra et al., 2010) or PHOENIX RapidFire (Chong et al., 2013) use a semi- empirical model that treats the ROS as a parametric function of biomass fuel properties, terrain topography and meteorological conditions; for instance, FARSITE uses a semiempirical model due to Rothermel (1972), while FOREFIRE is based on the quasi-physical model due to Balbi et al. (2009); a detailed review of ROS models is provided in Sullivan (2009). One recent strategy to better account for time-varying weather conditions at regional scales consists of coupling a front-tracking simulator for surface fires with a meso-scale CFD atmospheric model for fire-induced atmospheric dynamics, see for instance WRF-Fire (Kochanski et al., 2013) or FOREFIRE-MESONH (Filippi et al., 2013). Still, many uncertainties remain due to simplifications in the description of the physics and to knowledge gaps in the description of environmental conditions and yet, errors in the properties of the biomass fuel or in the flame/wind interactions induce strong changes in the heat transfer from the flame to the vegetation and in the biomass fuel pyrolysis for instance. Thus, modeling errors as well as errors in the boundary conditions (e.g., biomass fuel properties, meteorological conditions and terrain topography) and initial conditions inevitably translate into errors in the simulated and forecast positions of the fire front.

For the wildfire spread simulation to be predictive and compatible with operational applications, the uncertainties in the ROS semi-empirical model must be quantified and reduced through a data assimilation (DA) methodology. The uncertainties inherent in wildfire spread modeling suggest the use of ensemble-based DA. Ensemble forecasts stochastically characterize the non-linear response of models to variations in the input data (Finney et al., 2011). Furthermore, DA integrates available fire sensor observations into a computer modeling tool to correct and optimize the model outputs and to thereby produce improved forecast capabilities. Since sharp variations of the wind direction can lead to significant spatial deformations of the propagating fireline and since a canyon terrain can lead to eruptive fire behavior with strong acceleration in the steepest upslope directions, a spatially distributed correction along the fireline is required to be able to track the time-evolving location of the fire front.

This study is an extension of our previous works presented in Rochoux et al. (2012, 2013a, b, 2014a), in which a prototype data-driven wildfire simulator was developed. The initial prototype featured the following main components: an Eulerian front-tracking solver combined with a model description of the local ROS proposed by Rothermel (1972); an assumed series of airborne or possibly spaceborne observations of the fire front location; and a DA algorithm relying on parameter estimation. The DA prototype based on an ensemble Kalman filter (EnKF) was successfully evaluated in the context of a controlled grassland fire experiment (Paugam et al., 2013) when applied for estimating the input parameters used in the Rothermel-based ROS model (e.g., the fuel moisture content, the fuel particle surface-to-volume ratio, the wind direction and magnitude), see for instance Rochoux 
et al. (2014a). It was found that data-driven simulations are capable of correcting inaccurate predictions of the fire front location and of providing an optimized forecast of the wildfire behavior; the quality of the forecast prediction capability being dependent on the temporal variability of the errors in the ROS model parameters. While these studies confirmed the potential of a DA strategy for improved wildfire spread predictions, the choice of a spatially uniform parameter estimation approach is considered questionable. While well-suited for statistically spatially homogeneous problems (i.e., problems in which corrections to the parameters of the ROS model can be applied uniformly, at reduced scale for instance), this choice is no longer adapted to more general wildfire problems in which biomass fuel, topographical and possibly meteorological properties exhibit arbitrary spatial variations. In order to provide a spatial correction along the fireline, one could foresee the extension to the estimation of spatially distributed biomass, topographical, and wind ROS model parameters. As this solution would be computationally prohibitive in the context of real-time forecast of wildfire behavior and inconsistent with the actual knowledge of environmental conditions (Rochoux, 2014), an alternative solution is proposed here.

The objective of the present study is to remove some of the main limitations in the initial design of our prototype data-driven wildfire simulator with an extension to the case of spatially varying biomass fuel and meteorological conditions. This extension is based on a change from a parameter estimation (PE) approach to a state estimation (SE) approach that consists of sequentially updating the model state using complete or incomplete observations of the fire front (see Fig. 1). This correction of the fire front location at the observation time provides a more reliable initial condition to the fire spread model, and allows to produce ensemble-based forecasts of the fire front location that are more consistent with the actual fire behavior. In contrast to the PE approach that only addresses uncertainties in the ROS model parameters, the SE approach accounts for multiple sources of uncertainties that are difficult to distinguish but that contribute to the uncertainties in the fire front location, i.e., uncertainties in the ROS model parameters but also in the ROS parameterization itself noted $f$ in Eq. (1):

$\mathrm{ROS} \equiv f$ (wind conditions, biomass fuel properties).

The uncertainties in the function $f$ are partly due to simplifications in the physics of propagating fires when building the relation between ROS and environmental conditions (e.g., heat transfer to biomass fuels, steady-state assumption, non-local effects).

This change to a SE approach was inspired in part by previous studies by Mandel and Beezley (2007) and Mandel et al. $(2008,2011)$ in which the control variable is the temperature state variable and is characterized by a bimodal probability density function (PDF) in the fire region (i.e., burning state or non-burning state). In order to satisfy the Gaus-

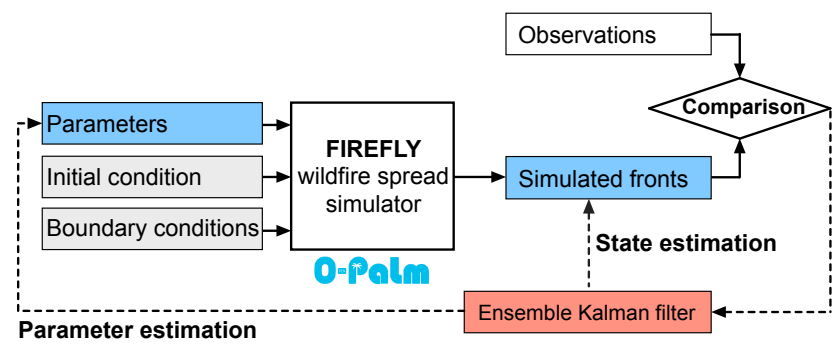

Figure 1. Data assimilation flowchart for PE and SE approaches over one assimilation cycle (control variables are colored in blue) using OpenPALM. Note that the SE approach provides the correction of the fire front location at the observation time and not at the initial time of the current assimilation cycle.

sian assumption in the EnKF, the idea of morphing from image processing was introduced (Mandel and Beezley, 2007); however, this choice led to technical difficulties in the EnKF implementation. In the present study, the fire front is represented as a finite number of front markers, which are equally spaced along the fireline that is projected onto the horizontal plane. Thus, the control vector includes the two-dimensional coordinates of each simulated front marker on the horizontal plane and is characterized by an approximate Gaussian PDF, which allows for a straightforward application of the EnKF. To obtain an efficient correction on the location of these front markers from a local observation, it is of primary importance to estimate reliable error correlations in the forecast error covariance matrix. Reliable means that these error correlations must be consistent with the actual spatial correlations of the errors in the fire spread model. For this purpose, an ensemble that accurately accounts for all sources of uncertainties must be generated during the EnKF prediction step.

This work provides valuable insight on how to best combine information from fire remote sensing and fire spread modeling using a data assimilation technique. Note that the validation of the prototype data-driven fire forecast system is currently limited to a reduced-scale controlled fire experiment. This system would need further developments to integrate the most recent progress in the fire radiative power (FRP) data analysis and in the fire spread modeling, and thereby directly address the operational requirements of wildfire spread monitoring.

The paper is organized as follows. The fire front observations and the wildfire spread model (called FIREFLY) are presented in Sect. 2. The EnKF algorithm is presented in Sect. 3. The performance of the resulting data-driven wildfire simulator is evaluated using first, academic tests in which observations are synthetically generated in Sect. 3; the focus is on the impact of ensemble generation on the representation of error correlations and on the resulting shape of the corrected fireline. The simulator performance is evaluated using second, a validation test in which observations are taken from a controlled grassland fire experiment in Sect. 4; a compar- 
ative study of the SE approach with the PE approach is also provided to highlight the benefits and drawbacks of each approach, in terms of update and forecast performances.

\section{Information on wildfires at regional scales: observations and forward model}

\subsection{Observations of the fire front location}

We assume in the present study that observations of the fire front location are available and that these observations can be made at different relevant times with a low measurement error. There is a growing body of literature on recent technological developments for geo-referenced wildfire front tracking (Riggan and Robert, 2009; Wooster et al., 2013), see Rochoux (2014) for a comprehensive review.

In the following, the observation vector noted $\boldsymbol{y}_{t}^{\mathrm{o}}$ contains the two-dimensional coordinates $\left(x_{i}^{\mathrm{o}}, y_{i}^{\mathrm{o}}\right)$ of the front markers at time $t$, with $i$ varying between 1 and $N_{\mathrm{fr}}^{\mathrm{o}}$. The coordinates of the fire front markers are assumed to have independent Gaussian-like random errors $\epsilon^{\mathrm{o}}$ with zero mean and with standard deviation (STD) noted $\sigma^{\circ}$. Two types of experiments are presented in this work: observation system simulation experiments (OSSEs), in which observations are synthetically generated using a reference (true) solution of the FIREFLY model that is modified by random observation errors $\epsilon^{\circ}$ (see Sect. 3); and a controlled grassland fire experiment, in which the observations are reconstructed from measured temperature maps and using a definition of the fire front as the $600 \mathrm{~K}$ iso-temperature contour (see Sect. 4).

\subsection{The fire spread model (the forward model)}

FIREFLY tracks the time-evolving location of the fire front using the following three components: (1) the Rothermelbased ROS model from the latest revision (Rothermel, 1972; Andrews et al., 2013):

$\Gamma \equiv \Gamma\left(\delta_{\mathrm{v}}, M_{\mathrm{v}}, M_{\mathrm{v}, \mathrm{ext}}, \Sigma_{\mathrm{v}}, m_{\mathrm{v}}^{\prime \prime}, \rho_{\mathrm{p}}, \Delta h_{\mathrm{c}}, u_{\mathrm{w}}\right)$,

where the nomenclature for the input parameters are summarized in Table 1; (2) an Eulerian front-tracking solver for the fire front propagation equation that computes the spatiotemporal variations of the progress variable $c \equiv c(x, y, t)$ introduced as a flame marker with $c=0$ in the unburnt vegetation, $c=1$ in the burnt vegetation, and where the flame front is identified as the contour line $c_{\mathrm{fr}}=0.5$; (3) an isocontour algorithm for the reconstruction of the fire front that discretizes the flame contour $c_{\mathrm{fr}}=0.5$ with $N_{\mathrm{fr}}$ markers, $\left(x_{i}, y_{i}\right)$ representing the two-dimensional coordinates of the $i$ th marker. Note that the wind velocity magnitude $u_{\mathrm{w}}$ is defined by the projection of the wind velocity vector (defined by its magnitude, $u_{\mathrm{w}}^{*}\left(\mathrm{~m} \mathrm{~s}^{-1}\right)$, and direction angle, $\alpha_{\mathrm{w}}^{*}\left({ }^{\circ}\right)$ ) along the (local) normal direction to the fire front, see Rochoux et al. (2014a) for further details on the front-tracking solver (e.g., numerical approach, model diagnostics).
Since its first developments (Rothermel, 1972), the basic mathematical formulation of the Rothermel model has been preserved. Users have adapted coefficients (such as the description of the fuel bed properties, see for instance Sandberg et al., 2007) and added optional submodels to fit specific cases of fire propagation. In this work, the latest revision from the Rothermel model (Andrews et al., 2013) is used (the original formulation includes a revised wind speed limit function to correct the rate of spread dependence on strong wind speeds). Since the focus is on the DA strategy, the complex description of the biomass fuel that can be used by forest service experts (Sandberg et al., 2007) is not addressed in this work. The default values given in Rothermel (1972) such as the moisture content at extinction $\left(M_{\mathrm{v}, \text { ext }}=30 \%\right)$ are therefore used.

Still, the PE and SE approaches presented in the following are valid for any fire spread model. While beyond the scope of this work, they could be extended to more complex description of the input data (biomass fuel, surface wind conditions, terrain topography) without changing the estimation algorithm. Furthermore, FIREFLY could readily be replaced by any other front-tracking simulator of wildfire spread, for instance FARSITE, FOREFIRE, PROMETHEUS or PHOENIX RapidFire.

The extension of FIREFLY to cases with complex terrain topography is outside the scope of the present study since the controlled grassland fire experiment used for the comparative evaluation of the PE and SE approaches corresponds to a reduced-scale, flat and horizontal, open-field grassland lot burning; a flat terrain is therefore assumed in both academic and validation tests presented in this paper.

\section{Data assimilation algorithm: specificities of the state estimation approach}

\subsection{Formulation of the ensemble Kalman filter}

We present here the ensemble Kalman filter (EnKF) algorithm applied, in the context of SE, for one assimilation cycle between time $(t-1)$ and time $t$; the PE approach is explained in detail in Rochoux et al. (2014a).

\subsubsection{Control space and observation space}

The DA algorithm uses a discretization of both the simulated and observed fire fronts, called SFF and OFF, respectively. The discretization of SFF is a set of $N_{\mathrm{fr}}$ markers; the control vector $\boldsymbol{x}_{t}$, also called the state vector, contains the twodimensional coordinates of the $N_{\mathrm{fr}}$ front markers at time $t$ :

$\boldsymbol{x}_{t}=\left[\left(x_{1}, y_{1}\right),\left(x_{2}, y_{2}\right) \ldots,\left(x_{N_{\mathrm{fr}}}, y_{N_{\mathrm{fr}}}\right)\right]$,

such that the size of $\boldsymbol{x}_{t}$ is $n=2 N_{\mathrm{fr}}$. 
Table 1. Input parameters of the Rothermel-based ROS sub-model.

\begin{tabular}{lcc}
\hline Name & Symbol & Unit \\
\hline Fuel depth (vertical thickness of the vegetation layer) & $\delta_{\mathrm{V}}$ & $\mathrm{m}$ \\
Fuel moisture (mass of water divided by mass of dry vegetation) & $M_{\mathrm{V}}$ & $\%$ \\
Fuel moisture at extinction & $M_{\mathrm{v}, \mathrm{ext}}$ & $\%$ \\
Fuel particle surface-to-volume ratio & $\Sigma_{\mathrm{V}}$ & $\mathrm{m}^{-1}$ \\
Fuel loading & $m^{\prime \prime \prime} / \mathrm{v}$ & $\mathrm{kg} \mathrm{m}^{-2}$ \\
Fuel particle mass density & $\rho_{\mathrm{p}}$ & $\mathrm{kg} \mathrm{m}^{-3}$ \\
Fuel heat of combustion & $\Delta h_{\mathrm{c}}$ & $\mathrm{J} \mathrm{kg}^{-1}$ \\
Wind velocity magnitude at mid-flame height (projected onto horizontal plane) & $u_{\mathrm{W}}$ & $\mathrm{m} \mathrm{s}^{-1}$ \\
\hline
\end{tabular}

Similarly, the discretization of OFF is a set of $N_{\mathrm{fr}}^{\mathrm{o}}$ markers; the observation vector $\boldsymbol{y}_{t}^{\mathrm{o}}$ of size $2 N_{\mathrm{fr}}^{\mathrm{o}}$ is defined as follows:

$\boldsymbol{y}_{t}^{\mathrm{o}}=\left[\left(x_{1}^{\mathrm{o}}, y_{1}^{\mathrm{o}}\right),\left(x_{2}^{\mathrm{o}}, y_{2}^{\mathrm{o}}\right), \ldots,\left(x_{N_{\mathrm{fr}}^{\mathrm{o}}}^{\mathrm{o}}, y_{N_{\mathrm{fr}}^{\mathrm{o}}}^{\mathrm{o}}\right)\right]$.

Observations of the fire front location are likely to be provided with a coarse resolution; in addition, observations may be incomplete and cover only a fraction of the fire front perimeter due to a limited monitoring or to the opacity of the fire-induced thermal plume. Thus, we may expect $N_{\mathrm{fr}}^{\mathrm{o}}$ to be much lower than $N_{\mathrm{fr}}$. In the following, we assume for simplicity that $N_{\mathrm{fr}}^{\mathrm{o}}=\left(N_{\mathrm{fr}} / r\right)$, where $r$ is an integer taking values (much) larger than 1 ( $r$ represents the difference in resolution between SFF and OFF).

\subsubsection{Observation operator}

In order to pair a subset of $N_{\mathrm{fr}}^{\mathrm{o}}$ markers along SFF with the $N_{\text {fr }}^{\mathrm{o}}$ markers along OFF, an observation operator $\mathcal{G}_{t}$ is introduced. Within the framework of SE, $\mathcal{G}_{t}$ is reduced to a selection operator $\mathcal{H}_{t}$ taking 1 out of every $r=\left(N_{\mathrm{fr}} / N_{\mathrm{fr}}^{\mathrm{o}}\right)$ markers along SFF at the observation time $t$. The model counterparts of the observation quantities, noted $\boldsymbol{y}_{t}$ and of size $2 N_{\mathrm{fr}}^{\mathrm{o}}$, read as follows:

$\boldsymbol{y}_{t}=\mathcal{G}_{t}\left(\boldsymbol{x}_{t}\right)=\mathcal{H}_{t}\left(\boldsymbol{x}_{t}\right)$

associating each marker of OFF with its closest neighbor along SFF. This representation of the fire fronts provides a local information efficient at retrieving the anisotropy in wildfire spread; the generalization of this representation to complex fire front topology will be addressed in future work.

\subsubsection{Sequential estimation}

The EnKF algorithm is sequentially applied; each assimilation cycle $[t-1, t]$ consists of two successive steps for each member of the ensemble indexed by the exponent $k$ as illustrated in Fig. 2:

1. a prediction step (forecast), in which the system is evolved from time $(t-1)$ to time $t$ ( $t$ being the next observation time) through an integration of FIREFLY to forecast the fire front location $\boldsymbol{x}_{t}$ given some uncertainty ranges in the ROS model parameters and in the fire ignition location $\left(x_{\text {ign }}, y_{\text {ign }}\right)$. This step leads to an ensemble of $N_{\mathrm{e}}$ fire front positions at time $t$ designated as follows:

$\boldsymbol{x}_{t}^{\mathrm{f}}=\left[\boldsymbol{x}_{t}^{\mathrm{f},(1)}, \cdots, \boldsymbol{x}_{t}^{\mathrm{f},(k)}, \cdots, \boldsymbol{x}_{t}^{\mathrm{f},\left(N_{\mathrm{e}}\right)}\right]$,

used to stochastically describe the error covariance matrix $\mathbf{P}_{t}^{\mathrm{f}} \in \mathbb{R}^{2 N_{\mathrm{fr}} \times 2 N_{\mathrm{fr}}}$ that is expressed as follows:

$\mathbf{P}_{t}^{\mathrm{f}}=\sum_{k=1}^{N_{\mathrm{e}}} \frac{\left(\boldsymbol{x}_{t}^{\mathrm{f},(k)}-\overline{\boldsymbol{x}_{t}^{\mathrm{f}}}\right)\left(\boldsymbol{x}_{t}^{\mathrm{f},(k)}-\overline{\boldsymbol{x}_{t}^{\mathrm{f}}}\right)^{T}}{N_{\mathrm{e}}-1}$,

where the overline denotes the mean value over the ensemble. The structure of $\mathbf{P}_{t}^{\mathrm{f}}$ is as follows:

- the first diagonal block (of size $N_{\mathrm{fr}} \times N_{\mathrm{fr}}$ ) represents the error covariances of the marker $x$ coordinates (univariate error covariances): in this block, each diagonal term represents the error variance of one marker $x$ coordinate, while off-diagonal terms represent the covariances of the error in one marker $x$ coordinate with the errors in the other marker $x$ coordinates;

- the second diagonal block (of size $N_{\mathrm{fr}} \times N_{\mathrm{fr}}$ ) represents the error covariances of the $y$ coordinates (univariate error covariances): in this block, each diagonal term represents the error variance of one marker $y$ coordinate, while off-diagonal terms represent the covariances of the error in one marker $y$ coordinate with the errors in the other marker $y$ coordinates;

- cross-diagonal blocks (of size $N_{\mathrm{fr}} \times N_{\mathrm{fr}}$ ) represent the (symmetric) multi-variate error crossedcovariances between the $x$ and $y$ coordinates of the $N_{\text {fr }}$ simulated front markers (multi-variate error covariances).

2. an update step (analysis), in which new observations $\boldsymbol{y}_{t}^{\mathrm{o}}$ are considered at the analysis time $t$ and in which the $k$ th 


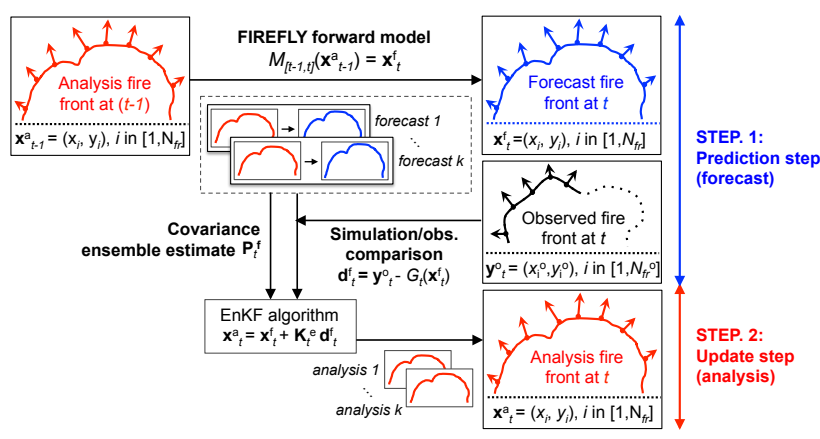

Figure 2. Flowchart of the EnKF algorithm during the $[t-1, t]$ assimilation cycle for a SE approach.

ensemble member is updated consistently with the observations, based on the following EnKF update equation:

$$
\begin{aligned}
& \boldsymbol{x}_{t}^{\mathrm{a},(k)}=\boldsymbol{x}_{t}^{\mathrm{f},(k)}+\mathbf{K}_{t}^{\mathrm{e}}\left(\boldsymbol{y}_{t}^{\mathrm{o}}+\boldsymbol{\xi}^{\mathrm{o},(k)}-\mathcal{H}_{t}\left(\boldsymbol{x}_{t}^{\mathrm{f},(k)}\right)\right), \\
& \mathbf{K}_{t}^{\mathrm{e}}=\mathbf{P}_{t}^{\mathrm{f}} \mathbf{H}_{t}^{\mathrm{T}}\left(\mathbf{H}_{t} \mathbf{P}_{t}^{\mathrm{f}} \mathbf{H}_{t}^{\mathrm{T}}+\mathbf{R}\right)^{-1},
\end{aligned}
$$

where, as for the PE approach (see Rochoux et al., 2014a), the innovation vector $\boldsymbol{d}_{t}^{(k)}$ is simply defined as the vector formed by the directed distances between the paired SFF-OFF markers for the $k$ th ensemble member:

$$
\boldsymbol{d}_{t}^{(k)}=\boldsymbol{y}_{t}^{\mathrm{o}}+\boldsymbol{\xi}^{\mathrm{o},(k)}-\boldsymbol{y}_{t}^{\mathrm{f}}=\boldsymbol{y}_{t}^{\mathrm{o}}+\boldsymbol{\xi}^{\mathrm{o},(k)}-\mathcal{H}_{t}\left(\boldsymbol{x}_{t}^{\mathrm{f},(k)}\right),
$$

with an additional noise $\xi^{\mathrm{o},(k)}$ added to the observation vector to avoid ensemble collapse (Burgers et al., 1998). The EnKF update in Eqs. (5) and (6) provides corrected positions $\boldsymbol{x}_{t}^{\mathrm{a}}$ for the $N_{\mathrm{fr}}$ simulated markers along the fireline at time $t$, but there is no feedback on the ROS model parameters and/or on the fire's initial location used to generate variability in the ensemble of forecasts. Note that in the context of SE, the selection operator $\mathcal{H}_{t}$ is straightforward to compute, meaning that a selection of lines and columns of $\mathbf{P}_{t}^{\mathrm{f}}$ is sufficient to estimate the term $\left(\mathbf{H}_{t} \mathbf{P}_{t}^{\mathrm{f}} \mathbf{H}_{t}^{\mathrm{T}}\right)$. Note also that in the present study as in Rochoux et al. (2014a), observation errors are assumed uncorrelated, i.e., the observation error covariance matrix $\mathbf{R}$ is treated as a diagonal matrix, in which each diagonal term is the error variance $\left(\sigma^{\circ}\right)^{2}$ associated with the $x$ or $y$ coordinate of the $N_{\mathrm{fr}}^{\mathrm{o}}$ markers along OFF.

\subsection{Ensemble generation}

The generation of the forecast ensemble is of primary importance in the performance of the EnKF forecast/update steps since it directly impacts how uncertainties are represented in the forecast error covariance matrix $\mathbf{P}_{t}^{\mathbf{f}}$. To allow for reliable error covariance modeling, all possible sources of uncertainties must be accounted for during this forecast step.
Different techniques can be considered to generate the ensemble of forecasts. If each fire front marker is perturbed separately (meaning that the error of one marker is uncorrelated to the errors of its neighbors along the fireline), the resulting fire front does not exhibit coherent features. This ensemble generation is therefore conflicting with the physical processes underlying wildfire spread. An alternative and more physically consistent strategy is to generate an ensemble of simulated fire fronts by (1) randomly perturbing the input parameters of the Rothermel-based ROS model (e.g., wind magnitude $u_{\mathrm{w}}^{*}$ and direction angle $\alpha_{\mathrm{w}}^{*}$, fuel moisture content $\left.M_{\mathrm{v}}\right)$ as well as the fire's initial location $\left(x_{\mathrm{ign}}, y_{\mathrm{ign}}\right)$, and by (2) integrating FIREFLY using the PALM-PARASOL functionality in OpenPALM ${ }^{1}$ (Fouilloux et al., 1999; Lagarde et al., 2001; Buis et al., 2006) for each set of parameters as in the PE approach, see Rochoux et al. (2014a) for details on PALM-PARASOL. This leads to an ensemble of $N_{\mathrm{e}}$ fire front locations at time $t$ designated as $\boldsymbol{x}_{t}^{\mathrm{f}}$ and used to describe the forecast error covariance matrix $\mathbf{P}_{t}^{f}$.

A series of OSSE tests cases is presented here to highlight the key aspects of the proposed SE approach. The focus is on the impact of ensemble generation on the stochastic estimation of the spatial correlations of the marker location errors in $\mathbf{P}_{t}^{\mathbf{f}}$ and the resulting correction of the fireline. Observation errors are assumed to be small with respect to the fireline perimeter (with the observation error STD taken equal to $\sigma^{0}=1 \mathrm{~m}$ ); the SE-based EnKF performance is evaluated by its ability to track the time-evolving location of the observed fire front.

\subsubsection{Isotropic case}

An isotropic case corresponding to a $200 \mathrm{~m} \times 200 \mathrm{~m}$ domain with uniform biomass fuel properties and no wind is considered first. The ROS $\Gamma$ is constant, uniform and taken equal to $0.2 \mathrm{~m} \mathrm{~s}^{-1}$. The true fire is ignited at $\left(x_{\mathrm{ign}}, y_{\mathrm{ign}}\right)=$ $(100 \mathrm{~m}, 100 \mathrm{~m})$ as a circular front with a radius of $5 \mathrm{~m}$. Using a $1 \mathrm{~m}$ computational grid resolution along the $x$ and $y$ directions and a $0.5 \mathrm{~s}$ temporal resolution, FIREFLY is first integrated over the time window $[0 ; 200 \mathrm{~s}]$ in order to produce at the analysis time (chosen to be $t=200 \mathrm{~s}$ ) the true location of the fire front. A forecast ensemble of $N_{\mathrm{e}}=25$ members is then produced based on spatial variations of the fire's initial location $\left(x_{\mathrm{ign}}, y_{\mathrm{ign}}\right)$ around a mean value $(97 \mathrm{~m}$, $103 \mathrm{~m}$ ) and with an error STD along both $x$ and $y$ directions taken equal to $\sigma^{f}=10 \mathrm{~m}$, see Fig. 3a. In this test, uncertainties in the forecast ensemble are only due to errors in the initial location of the fire front. The observed fire front is described by a stand-alone marker $\left(N_{\mathrm{fr}}^{\mathrm{o}}=1\right)$, while simulated fire fronts are discretized using $N_{\text {fr }}=100$ markers (i.e., $r=N_{\mathrm{fr}} / N_{\mathrm{fr}}^{\mathrm{o}}=100$ ).

Figure 3 a presents a comparison between the true and forecast fire front positions at time $t=200$ s (i.e., the observation

\footnotetext{
${ }^{1}$ http://www.cerfacs.fr/globc/PALM_WEB/
} 


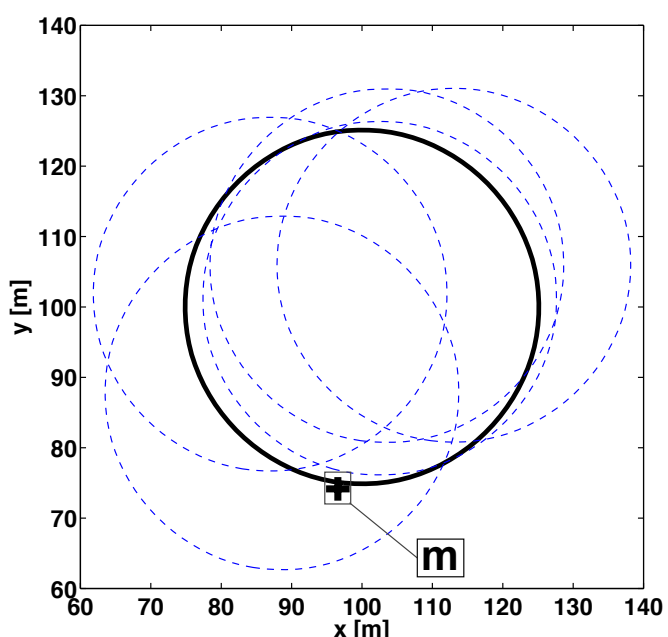

(a) Forecast estimates of the fire front location

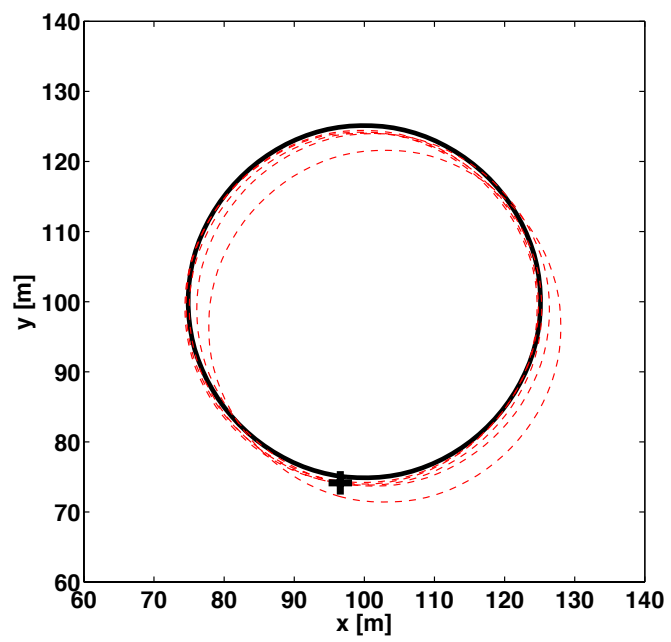

(b) Analysis estimates of the fire front location

Figure 3. Spatially uniform OSSE test with constant ROS but uncertain ignition location $\left(x_{\text {ign }}, y_{\text {ign }}\right)$; one assimilation cycle [0; $200 \mathrm{~s}$ ]; all figures correspond to the observation time $t=200 \mathrm{~s}$. (a) Comparison between true (black solid line) and forecast (blue dashed lines) fire front positions; the cross symbol is the only observation available. (b) Similar comparison between true (black solid line) and analysis (red dashed lines) fire front positions.

time). This figure shows that due to uncertainties in the initial location of the fire front at initial time $(0 \mathrm{~s})$, the predicted fire front locations are scattered over a large area at observation time (200s). Since in this test, uncertainties in the distribution of the biomass fuel properties are not accounted for in the ensemble generation, the propagation of the fire front is isotropic (simulated fire fronts remain circular).

Figure 4 presents the spatial correlation of the error in the location of the marker indexed by $\mathrm{m}$ in Fig. 3a. Note that the error correlation associated with the marker $m$ represents how the error in the location of this simulated marker is correlated to the errors in the location of its neighbors along the

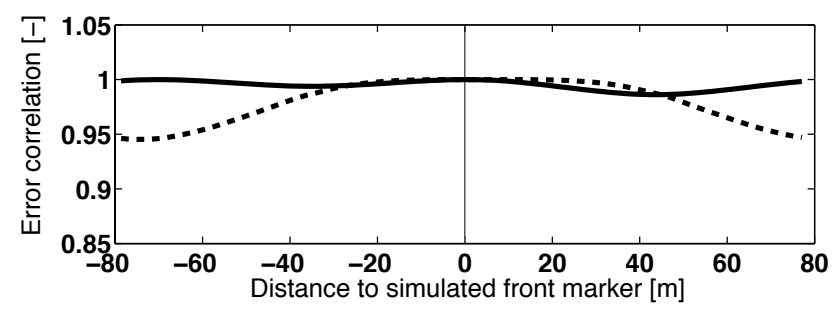

(a) Univariate error correlations along the fireline

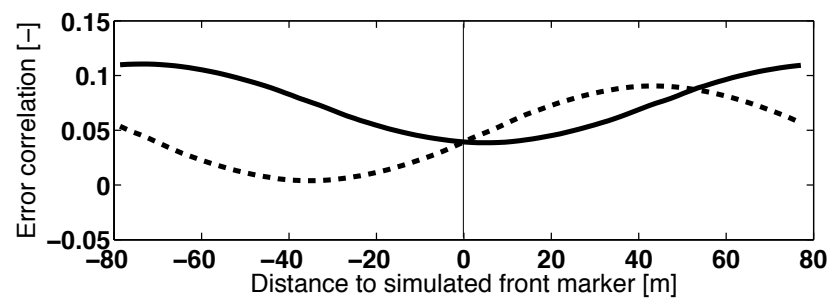

(b) Multi-variate error correlations along the fireline

Figure 4. Error correlation functions along the fireline associated to one marker location (the vertical bar represents its reference location indexed by $\mathrm{m}$ in Fig. 3a) for the OSSE isotropic propagation test with constant ROS but uncertain ignition location $\left(x_{\text {ign }}, y_{\text {ign }}\right)$ at the observation time $t=200 \mathrm{~s}$. (a) Univariate correlations: the dashed (solid) line indicates the error correlation of the reference marker $x$ coordinate ( $y$ coordinate) with respect to the errors in the $x$ coordinates ( $y$ coordinates) of the other markers along the fireline. (b) Multi-variate correlations: the dashed (solid) line indicates the error correlation of the reference marker $x$ coordinate ( $y$ coordinate) with respect to the errors in the $y$ coordinates ( $x$ coordinates) of the other markers along the fireline.

fireline (this, in order to characterize the fire front as a coherent feature given by FIREFLY). Figure 4 shows that univariate correlations are almost equal to one, while multi-variate correlations are nearly zero. Errors in the position of the $N_{\mathrm{fr}}$ simulated front markers are highly correlated within the ensemble due to the isotropic propagation. As a result, the DA algorithm translates the information observed at one marker into a uniform correction along the fireline.

Figure $3 \mathrm{~b}$ presents the comparison between the true and analysis fire front positions at the observation time $t=200 \mathrm{~s}$. The analysis ensemble corresponds here to the updated fire front locations that are produced through the EnKF update step. As expected, the analysis estimates of the fire front locations feature a much reduced scatter around the true location of the fire front, and the EnKF correction is isotropic due to high error correlations along the fireline (i.e., each analysis estimate within the ensemble is a circular front). With this uniform definition of the forecast ensemble, deforming the shape of the fire front through the ensemble-based analysis is impossible. 


\subsubsection{Anisotropic case}

To be able to stochastically represent more complex front shapes that are representative of all sources of uncertainties in the wildfire spread model as well as of their spatial variability, it is of primary importance to consider non-uniform environmental conditions when generating the ensemble of forecasts.

An anisotropic case of wildfire spread subject to spatially varying vegetation properties and wind-aided propagation is now considered. An ensemble of $N_{\mathrm{e}}=20$ forecasts is produced over the time window $[0 ; 150 \mathrm{~s}]$, based on assumed uncertainties in the fire ignition location $\left(x_{\mathrm{ign}}, y_{\mathrm{ign}}\right)$ at initial time $(0 \mathrm{~s})$ as well as in a subset of ROS model parameters, specifically in the fuel layer depth $\delta_{\mathrm{V}}$, the fuel moisture content $M_{\mathrm{v}}$, the fuel particle surface-to-volume ratio $\Sigma_{\mathrm{v}}$ and the wind properties (magnitude $u_{\mathrm{w}}^{*}$ and direction angle $\alpha_{\mathrm{w}}^{*}$ ). In addition, the fuel depth $\delta_{\mathrm{v}}$ is assumed to be spatially varying, taking different values in the four quadrants of the square-shaped $700 \mathrm{~m} \times 700 \mathrm{~m}$ computational domain. Thus, uncertainties in the forecast ensemble are due to variations in 10 parameters, whose mean and STD values are presented in Table 2. Simulated fire fronts are discretized using $N_{\text {fr }}=100$ markers; the impact of spatial error correlations on the EnKF-based analysis estimates is studied here for varying number of observed front markers $N_{\mathrm{fr}}^{\mathrm{o}}$.

Figure 5a presents a comparison between the true and forecast fire front positions at observation time $t=150 \mathrm{~s}$. Due to uncertainties in the ROS model parameters and not only uncertainties in the fire's initial location, the propagation is now anisotropic; due to the presence of wind and to the spatial variations in fuel depth, the forecast fire fronts are characterized by stronger irregularities and more complex shapes than results presented in Fig. 3a. Figure 5b presents a similar comparison between the true and analysis fire front positions at time $t=150 \mathrm{~s}$; the observed fire front is described by $N_{\mathrm{fr}}^{\mathrm{o}}=20$ uniformly distributed markers (i.e., $r=5$ ). As expected, the ensemble of analyses features a much reduced scatter (in terms of front shapes) around the true location of the fire front.

While Fig. 5a-b shows that the direct observation of the fire front location can overcome various uncertainties in the ROS model parameters, Fig. 5c illustrates that the spatial distribution of the observations along the fireline has a significant impact on the analysis. This figure considers a practically relevant situation in which the observations are limited to a certain section of the fireline (i.e., the informed section, possibly due to the opacity of the thermal plume) and therefore, provide an incomplete picture. In this situation, while the EnKF algorithm produces an analysis that is close to the true state in the informed section, the benefits of DA are reduced in the non-informed sections. However, in spite of a reduced level of performance, the EnKF algorithm remains capable of a significant improved performance compared to a stand-alone forecast (in terms of front shapes and loca-

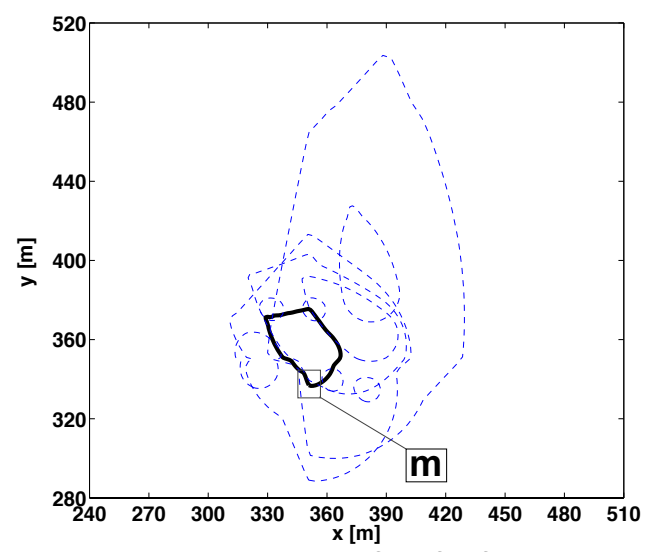

(a) Forecast estimates of the fire front location

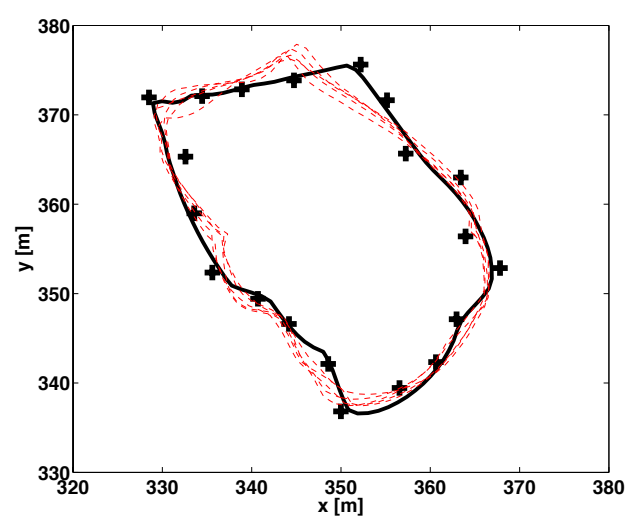

(b) Analysis estimates of the fire front location with $N_{\mathrm{fr}}{ }^{\circ}=20$

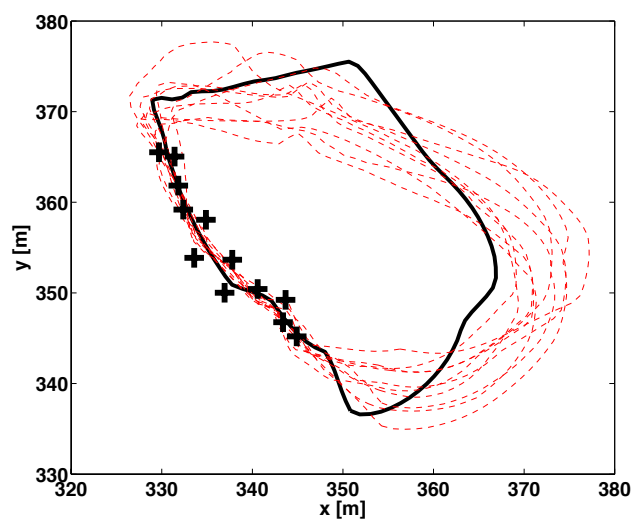

(c) Analysis estimates of the fire front location with $N_{\mathrm{fr}}{ }^{\circ}=12$ for an incomplete set of observations

Figure 5. Spatially varying OSSE test with uncertain ROS model parameters and with uncertain ignition location $\left(x_{\text {ign }}, y_{\text {ign }}\right)$; one assimilation cycle $[0 ; 150 \mathrm{~s}]$; all figures correspond to the observation time $t=150 \mathrm{~s}$. (a) Comparison between true (black solid line) and forecast (blue dashed lines) fire front positions. (b-c) Comparison between true (black solid line) and analysis (red dashed lines) fire front positions. Black cross symbols are the $N_{\mathrm{fr}}^{\text {os }}$ observed front markers. 
Table 2. Properties of the ensemble forecast in the OSSE anisotropic case.

\begin{tabular}{lrrr}
\hline Random variable & True value & Ensemble mean & Ensemble STD \\
\hline$\left(\delta_{\mathrm{v}, 1}, \delta_{\mathrm{v}, 2}, \delta_{\mathrm{v}, 3}, \delta_{\mathrm{v}, 4}\right)(\mathrm{m})$ & $(0.25,1.25,0.75,1.75)$ & $(0.25,1.25,0.75,1.75)$ & $(0.10,0.10,0.10,0.10)$ \\
$M_{\mathrm{V}}(\%)$ & 20 & 20 & 10 \\
$\Sigma_{\mathrm{V}}\left(\mathrm{m}^{-1}\right)$ & 11500 & 11500 & 4000 \\
$\left(u_{\mathrm{W}}^{*}, \alpha_{\mathrm{W}}^{*}\right)\left(\mathrm{m} \mathrm{s}^{-1},{ }^{\circ}\right)$ & $(1.0,315)$ & $(0.75,315)$ & $(0.15,45)$ \\
$x_{\mathrm{ign}}(\mathrm{m})$ & 350 & 350 & 20 \\
$y_{\text {ign }}(\mathrm{m})$ & 350 & 350 & 20 \\
\hline
\end{tabular}

tions). This improved performance is due to the spatial correlation of the errors in the location of the simulated markers. Since the anisotropy of wildfire spread is now represented by a wide range of uncertainties in the forecast ensemble, the error in the location of one observed marker is correlated with the error in the location of its adjacent markers along the fireline as shown in Fig. 6 for the marker indexed by $m$ in Fig. 5 a. Stated differently, the estimation problem translates the information coming from one observation marker into a local correction restricted to the closest neighbors only. The distance over which the observation marker affects the correction of the simulated front marker locations is referred to as correlation length scale (Daley, 1991; Pannekoucke et al., 2013). This length scale depends on the spatial variability of the errors in the ensemble generation and on the consistency of these statistics with the model errors statistics. For instance, Fig. 6 shows that the length scale associated with the univariate error correlations of the marker $x$ coordinate typically takes values on the order of $15 \mathrm{~m}$ on both sides of the considered simulated marker. This means that if this marker is assimilated, the correction of its location subsequently modifies the shape of the fire front over a distance of $15 \mathrm{~m}$ on both sides of this marker. This figure also shows that the errors in the marker $y$ coordinates are anti-correlated on both sides of the marker m; due to its particular location on the fireline (i.e., at the boundary between the flank and the back side of the fire), variations in the wind conditions induce significant changes in the fire front shape in the vicinity of marker $m$ (markers can move from the flank to the back side of the fire or from the back side to the flank by modification of the wind direction for instance). As a consequence, when several observations are available, a non-uniform correction is obtained, and the DA algorithm is able to change the shape of the analysis fronts and more easily match the observations.

Figure 7 examines the influence of the number of uniformly distributed markers along the observed fire front $N_{\mathrm{fr}}^{\mathrm{o}}$ on the EnKF performance. This figure presents the root mean square (RMS) distance between the true and the forecast fire front positions as well as between the true and the analysis fire front positions as a function of $N_{\mathrm{fr}}^{\mathrm{o}}$. The figure shows that when $N_{\mathrm{fr}}^{\mathrm{o}}$ is large (see Fig. $5 \mathrm{~b}$ with $N_{\mathrm{fr}}^{\mathrm{o}}=20$ ), the EnKF algorithm successfully drives the analysis ensemble towards

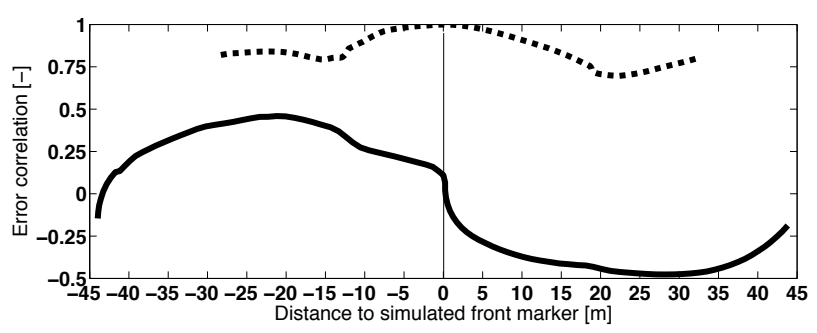

(a) Univariate error correlations along the fireline

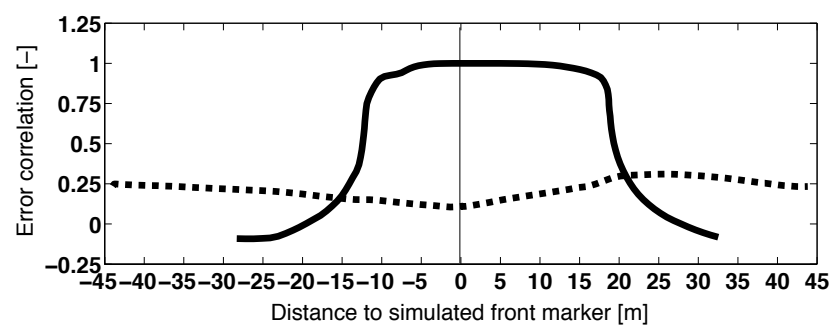

(b) Multi-variate error correlations along the fireline

Figure 6. See caption of Fig. 4. OSSE anisotropic case with uncertain ROS model parameters and with uncertain ignition location $\left(x_{\text {ign }}, y_{\text {ign }}\right)$ at the observation time $t=150 \mathrm{~s}$; the vertical bar represents its reference location indexed by $\mathrm{m}$ in Fig. 5a.

the true state; in contrast, when $N_{\mathrm{fr}}^{\mathrm{o}}$ is small, the EnKF algorithm has reduced effects and the analysis estimates remain close to the forecast estimates. Stated differently, the performance of the DA algorithm and its ability to capture the highresolution features of wildfire spread depend strongly on the density of the observation network.

Furthermore, even though accounting for a wide range of uncertainties in the ROS parameters provides a wide range of possible fire front shapes, sampling errors can degrade the representation of the error statistics during the EnKF prediction step (if the number of members $N_{\mathrm{e}}$ in the ensemble is not large enough) and thereby, the analysis solution. Figure 8 examines the impact of $N_{\mathrm{e}}$ on the EnKF performance, in terms of the RMS distance between the true and forecast fire front positions as well as between the true and analysis fire front positions. These results show that for the present anisotropic case, $N_{\mathrm{e}}=20$ members are sufficient to obtain converged error statistics; below this threshold value, sampling noise in- 


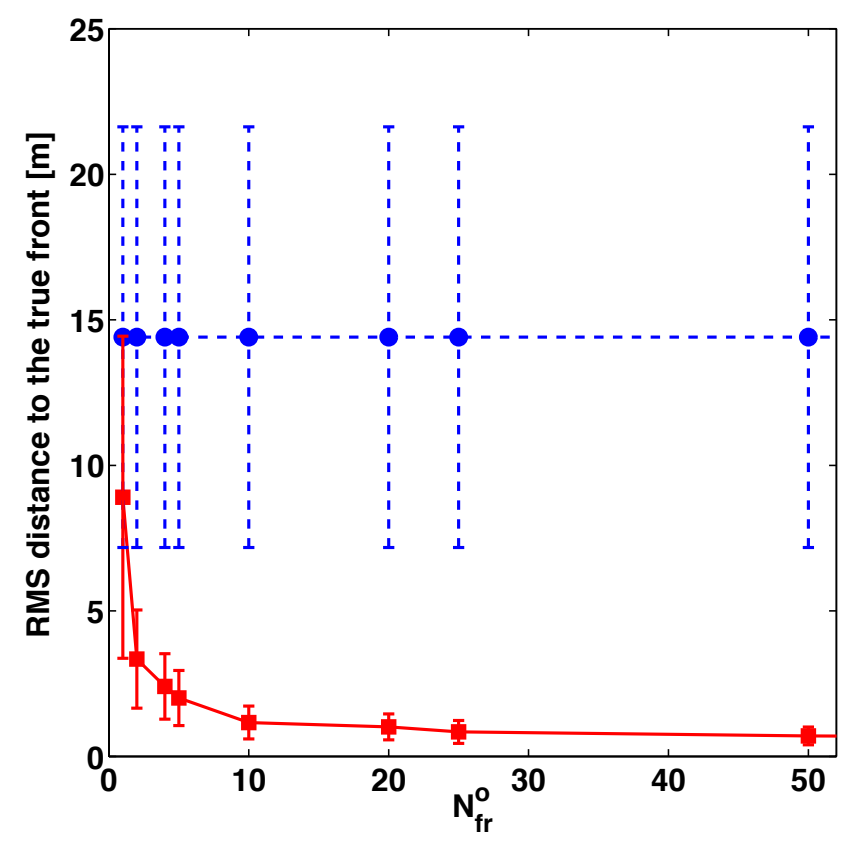

Figure 7. Average distance between the true and forecast fire front positions (blue circles) and between the true and analysis fire front positions (red squares) at time $150 \mathrm{~s}$ as a function of the number of observed markers $N_{\mathrm{fr}}^{\mathrm{os}}$; OSSE anisotropic case. Error bars indicate the associated STD.

duces significant errors in the representation of error covariances.

\subsection{Sequential model state estimation}

\subsubsection{Principle and algorithm}

To apply successive assimilations (i.e., perform regular EnKF updates), the ensemble of analysis fire fronts must be used as initial conditions and evolved to future times. However, to restart the FIREFLY front-tracking simulator, a two-dimensional progress variable field $c$ is required (see Sect. 2.2). The field $c^{(k)} \equiv c(x, y, t)^{(k)}$ associated with the $k$ th ensemble member $\boldsymbol{x}_{t}^{a,(k)}$ ( $k$ varying between 1 and $N_{\mathrm{e}}$ ) is therefore reconstructed and used as initial condition for the next assimilation cycle $[t, t+1]$. This reconstruction is performed through a binarization process, i.e., $c^{(k)}=0$ in the unburnt vegetation and $c^{(k)}=1$ in the burnt area.

For each member $k$ in the ensemble, the reconstruction algorithm applied to each mesh node $\left(x_{N}, y_{N}\right)$ of the computational domain is as follows (see Fig. 9):

1. pair the mesh node $\left(x_{N}, y_{N}\right)$ with the closest simulated front marker noted $\left(x_{A}, y_{A}\right)$ (computation of the minimal distance to the fire front);

2. determine the closest neighbor of $\left(x_{A}, y_{A}\right)$ along the fireline noted $\left(x_{B}, y_{B}\right)$;

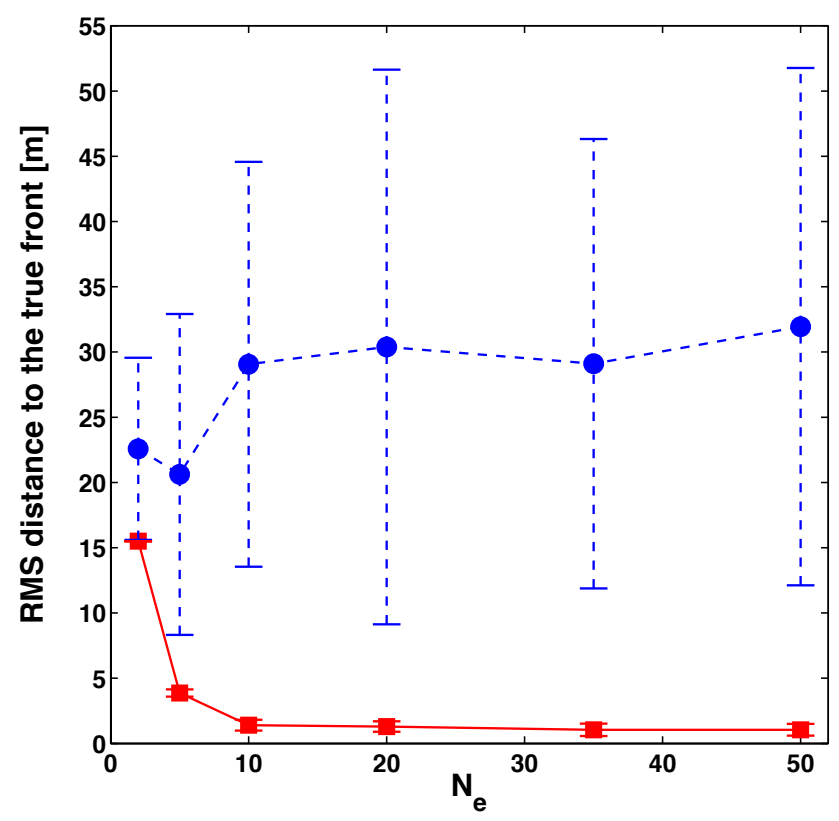

Figure 8. Average distance between the true and forecast fire front positions (blue circles) and between the true and analysis fire front positions (red squares) at time $150 \mathrm{~s}$ as a function of the number of members $N_{\mathrm{e}}$ in the ensemble; OSSE anisotropic case. Error bars indicate the associated STD.

3. compute the normal vector to the fireline (orthogonal to segment $[A B])$ passing through the node $\left(x_{N}, y_{N}\right)$, and determine their intersection noted $\left(x_{O}, y_{O}\right)$ (by linear algebra);

4. calculate the two-dimensional inner product between the vector $\overrightarrow{\boldsymbol{O N}}$ and the outward normal vector to the front at node $\left(x_{A}, y_{A}\right)$ noted $\overrightarrow{\boldsymbol{n}_{A}}$ : the sign of the inner product determines if the mesh node $\left(x_{N}, y_{N}\right)$ is inside or outside the burnt area:

- if the inner product $\left(\overrightarrow{\boldsymbol{O N}} \cdot \overrightarrow{\boldsymbol{n}_{A}}\right)$ is positive, the mesh node $\left(x_{N}, y_{N}\right)$ is outside the burnt area;

- if $\left(\overrightarrow{\boldsymbol{O N}} \cdot \overrightarrow{\boldsymbol{n}_{A}}\right)$ is negative, the mesh node $\left(x_{N}, y_{N}\right)$ is within the burnt area;

- if $\left(\overrightarrow{\boldsymbol{O N}} \cdot \overrightarrow{\boldsymbol{n}_{A}}\right)$ takes a zero value, the mesh node $\left(x_{N}, y_{N}\right)$ is on the fireline.

5. Determine the corresponding value of the progress variable $c$ at mesh node $\left(x_{N}, y_{N}\right)$; a smoothing hyperbolic tangent function is applied when the mesh node $\left(x_{N}, y_{N}\right)$ is near the fire front.

The reconstructed progress variable field $c^{(k)}$ can be used to integrate FIREFLY over the next forecast period $[t ; t+1]$.

A schematic of the sequential SE approach over the assimilation cycle $[t-1, t]$ for the $k$ th ensemble member is presented in Fig. 10; the main steps are as follows: 


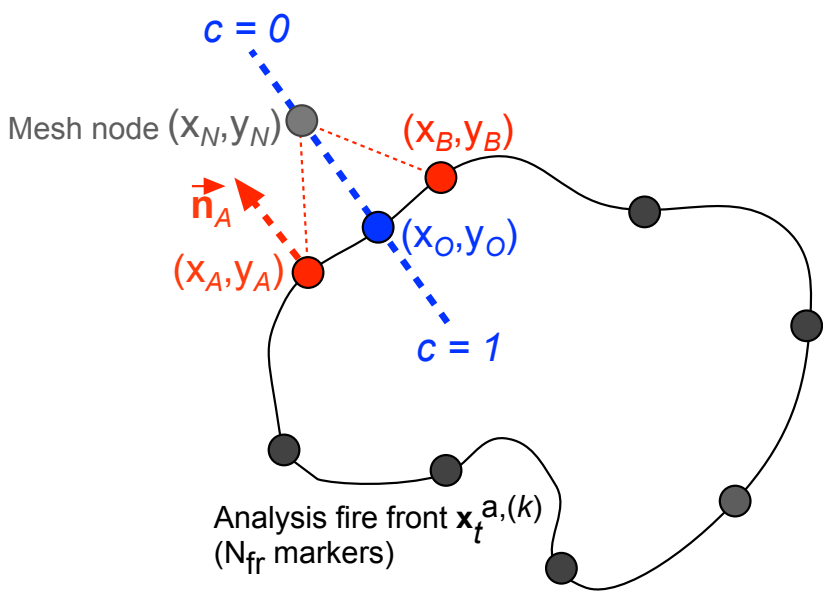

Figure 9. Schematic of the reconstruction algorithm for the FIREFLY initial condition. In this example, the mesh node $\left(x_{N}, y_{N}\right)$ is outside the burnt area, the inner product $\left(\overrightarrow{\boldsymbol{O N}} \cdot \overrightarrow{\boldsymbol{n}_{A}}\right)$ is positive.

1. build an ensemble of perturbed ROS model parameters (see Sect. 3.2);

2. integrate the forward model from time $(t-1)$ to time $t$, starting from the analysis progress variable field related to the $k$ th member at time $(t-1)$ and using the set of perturbed parameters related to the $k$ th member;

3. compute the forecast error covariance matrix $\mathbf{P}_{t}^{\mathrm{f}}$ using Eq. (4);

4. compute the observation operator reduced to a selection operator through Eq. (3), in order to obtain the model counterparts of the observations at time $t$;

5. apply the Kalman filter update equation at time $t$ for each member of the ensemble based on Eqs. (5)-(6), in order to obtain the corrected positions of the front markers at time $t$;

6. reconstruct the progress variable two-dimensional field $c^{(k)}$ corresponding to the analysis estimates at time $t$ (the reconstruction procedure must be performed for all members $N_{\mathrm{e}}$ in the ensemble).

To move to the next assimilation cycle $[t, t+1]$, step (1) can be performed again.

\subsubsection{Sequential assimilation for the anisotropic case}

Multiple assimilation cycles for the OSSE anisotropic case (presented in Sect. 3.2.2) are now considered based on the previously described multi-cycle algorithm. The quality of the forecast (measured by its ability to track the location of the true fire front) is examined over the time window $[0 ; 600 \mathrm{~s}]$ with observations taken at $150 \mathrm{~s}$ time intervals, at times $t_{1}=150 \mathrm{~s}, t_{2}=300 \mathrm{~s}, t_{3}=450 \mathrm{~s}$ and $t_{4}=600 \mathrm{~s}$. Thus,

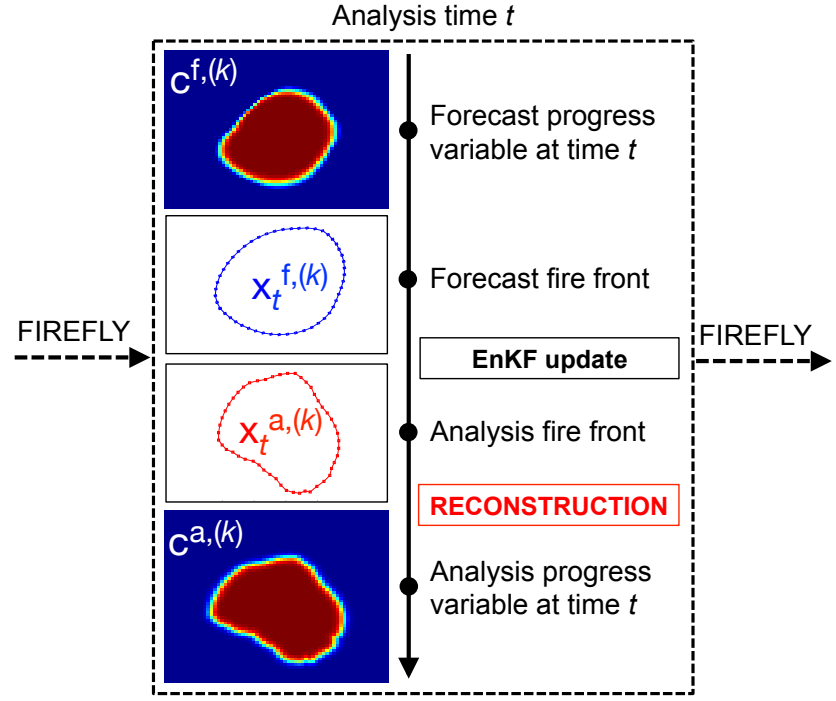

Figure 10. Schematic of the sequential SE approach for each ensemble member $k=1, \cdots, N_{\mathrm{e}}$, with a focus on the reconstruction of the two-dimensional progress variable $c^{(k)}$ posterior to analysis over the time window $[t-1, t]$ and prior to forecast over the time window $[t, t+1]$.

Table 3. Time-varying true wind conditions (in terms of magnitude $u_{\mathrm{w}}^{*}$ and direction angle $\alpha_{\mathrm{w}}^{*}$ ) in the OSSE anisotropic case with multiple assimilation cycles, from 0 to $600 \mathrm{~s}$, at $150 \mathrm{~s}$ time intervals.

\begin{tabular}{lll}
\hline $\begin{array}{l}\text { Assimilation } \\
\text { cycle }\end{array}$ & $u_{\mathrm{w}}^{*}\left(\mathrm{~m} \mathrm{~s}^{-1}\right)$ & $\alpha_{\mathrm{w}}^{*}\left(^{\circ}\right)$ \\
\hline 1 & 1.0 & 315 \\
2 & 0.75 & 290 \\
3 & 0.83 & 257 \\
4 & 1.20 & 232 \\
\hline
\end{tabular}

the EnKF update is performed over four successive assimilation cycles; each assimilation cycle $\left[t_{n-1} ; t_{n}\right]$ includes a prediction step that integrates FIREFLY from $t_{n-1}$ until $t_{n}$ and an update step that corrects the location of the simulated fire front at time $t_{n}(n=1, \ldots, 4)$. The propagation of the true fire front is simulated for time-varying wind conditions (but with assumed constant wind velocity and direction over each assimilation cycle) presented in Table 3 , while the forecast ensemble is simulated for constant wind conditions using the ROS model parameters presented in Table 2. Note that the perturbation of the fire ignition location $\left(x_{\mathrm{ign}}, y_{\mathrm{ign}}\right)$ is only introduced during the first assimilation cycle (as a means to account for uncertainties in the fire's initial location before remote sensing detection).

The quality of the forecast is expected to deteriorate at increasing lead-times (i.e., when the time delay between the actual forecast time and the previous analysis time increases) for two reasons. First, because the impact of the fire front correction applied at a given analysis time decreases with 

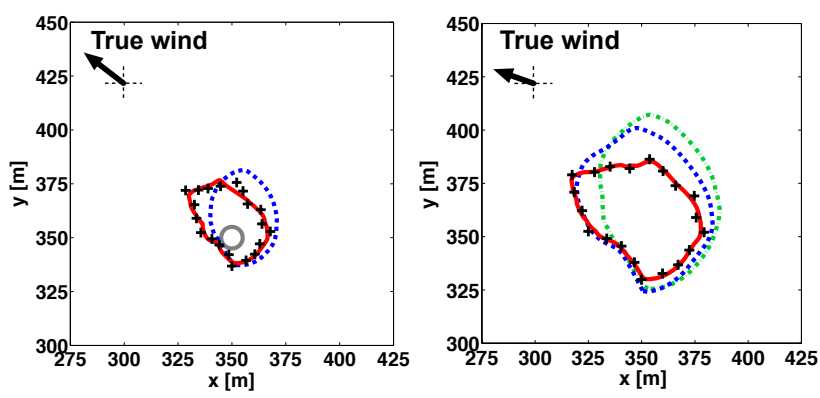

(a) $t_{1}=150 \mathrm{~s}$

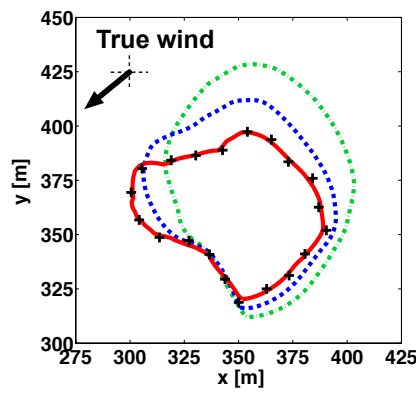

(c) $t_{3}=450 \mathrm{~s}$ (b) $\mathrm{t}_{2}=300 \mathrm{~s}$

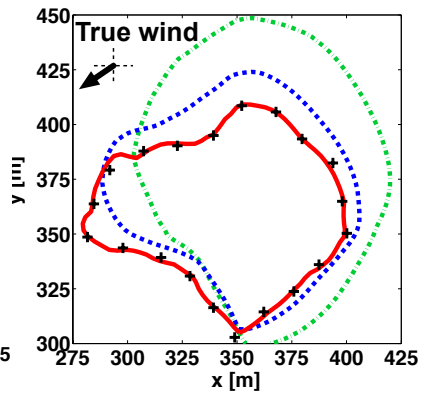

(d) $\mathrm{t}_{4}=600 \mathrm{~s}$

Figure 11. OSSE anisotropic case over multiple assimilation cycles (from 0 to $600 \mathrm{~s}$ ): comparison between the free run (green dasheddotted line), the mean forecast estimate (blue dashed line), observations (black crosses) and the mean analysis estimate (red solid line) at $150 \mathrm{~s}$ time intervals, at times (a) $t_{1}=150 \mathrm{~s}$ (the gray circle corresponds to the true initial location); (b) $t_{2}=300 \mathrm{~s}$; (c) $t_{3}=450 \mathrm{~s}$; and (d) $t_{4}=600 \mathrm{~s}$

time (i.e., when the forecast lead-time increases). Second, because the present implementation of the SE-based EnKF does not provide any correction for ROS modeling errors or for ROS model parameters (including the incorrect assumption of a constant wind); such correction may be addressed through a PE approach, see Rochoux et al. (2014a).

Figure 11 presents the successive comparison between the mean free run (i.e., stand-alone FIREFLY simulation without DA as green dashed-dotted line), the mean fire front location related to the forecast estimates (blue dashed line) and to the analysis estimates (red solid line) as well as the observations (black crosses) at times $t_{1}, t_{2}, t_{3}$ and $t_{4}$. It is found that the free run simulation does not accurately estimate the rate and direction of the fire spread due to imperfect knowledge in the ROS model parameters and in the fire's initial location. As for the forecast, it provides a more accurate prediction of the fire front location and a more physically consistent front shape than the free run at each assimilation time. Still, the analysis estimates exhibit a much reduced scatter due to the EnKF update and the information gain obtained by the observations:; the distance between the analysis fire front and the observations is largely reduced at the analysis time, and the shape of the analysis fire front is much more consistent with the observed fire front.

The performance of the EnKF update is confirmed by the error statistics presented in Fig. 12a, which presents the timeevolution of the deviations of FIREFLY model predictions from observations. It is shown that the benefits from the EnKF update decrease as the forecast lead-time increases. For instance, the RMS distance to the true front is significantly reduced by the EnKF update, from $30 \mathrm{~m}$ for the free run (FR) to less than $1 \mathrm{~m}$ for the analysis (A1) during the first assimilation cycle $[0 ; 150 \mathrm{~s}]$. Starting again from the analysis estimates at time $150 \mathrm{~s}$, FIREFLY simulates the forecast fire evolution in time but, without additional observations, the distance between the true state and the forecast (F1) significantly increases, to $20 \mathrm{~m}$ at time $t_{2}=300 \mathrm{~s}$ up to approximately $80 \mathrm{~m}$ at time $t_{5}=750 \mathrm{~s}$. By repeating the EnKF update at $150 \mathrm{~s}$ time intervals, the distance between the true state and the simulated fire front remains below $10 \mathrm{~m}$. EnKF-based data-driven simulations bring valuable information on the wildfire spread behavior, even when DA is not applied systematically. This is illustrated in Fig. 12b, which presents a comparison of different fire front forecasts at time $t_{4}=600 \mathrm{~s}$ using FIREFLY, with or without DA. The closer the assimilation time to $t_{4}=600 \mathrm{~s}$, the better the forecast prediction; consistently, the free run (FR) provides the less accurate prediction with a mean distance to the observations approximately equal to $70 \mathrm{~m}$; in contrast, this mean distance is reduced by a factor of nearly 2 for (F2) and by a factor of 70 for (A4).

In summary, these results of OSSE test cases show that in a SE approach, EnKF updates provide valuable information and lead to accurate forecasts on wildfire behavior at short lead-times, the definition of short being dependent on the persistence of the model initial condition. Accordingly, the EnKF update must be performed at regular time intervals to efficiently track the actual wildfire propagation.

\section{Application to a controlled grassland fire experiment}

The EnKF-FIREFLY data-driven simulator is now evaluated in a validation study corresponding to a controlled grassland fire experiment (a case in which the true control vector is not known and may not exist if the model is not sufficiently representative). The experimental configuration corresponds to a small $(4 \mathrm{~m} \times 4 \mathrm{~m})$, flat and horizontal, open-field grassland lot burning under moderate wind conditions in which the ROS takes values on the order of $1 \mathrm{~cm} \mathrm{~s}^{-1}$ (with a maximum value reaching $5 \mathrm{~cm} \mathrm{~s}^{-1}$ in the wind direction).

The properties of the grass are (approximately) known: $\delta_{\mathrm{v}}=8 \mathrm{~cm}$ (field measurement), $M_{\mathrm{v}}=22 \%$ (field measurement) and $\Sigma_{\mathrm{v}}=11500 \mathrm{~m}^{-1}$ (Rothermel's fuel database for short grass); the wind conditions are also approximately known: the magnitude and direction angle of the wind are constant and equal to $u_{\mathrm{w}}^{*}=1 \mathrm{~m} \mathrm{~s}^{-1}$ and $\alpha_{\mathrm{w}}^{*}=307^{\circ}$. The fire spread is recorded using a thermal-infrared camera; the thermal maps are post-processed (the fire front is defined at the 


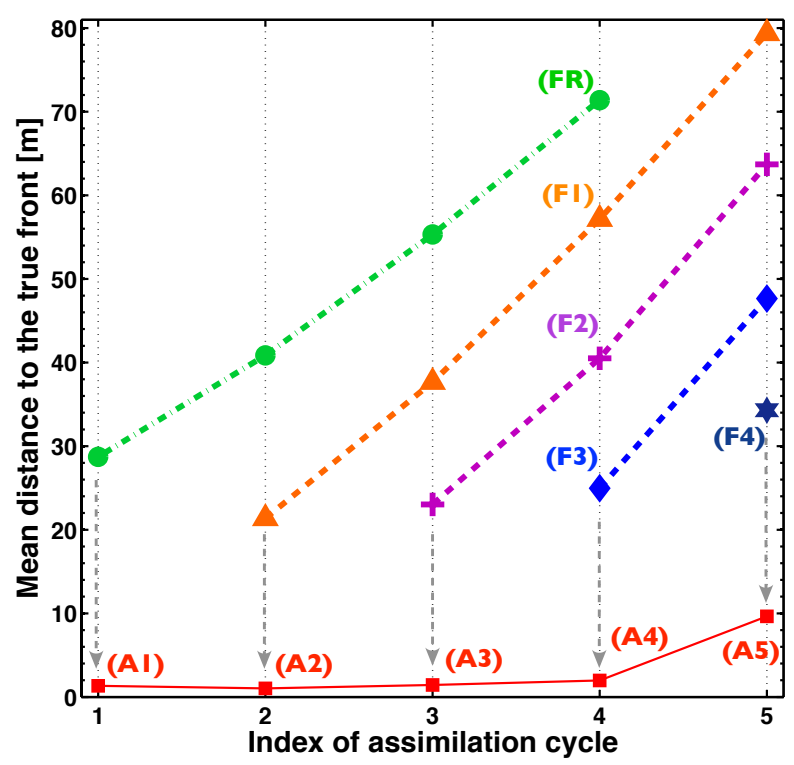

(a)

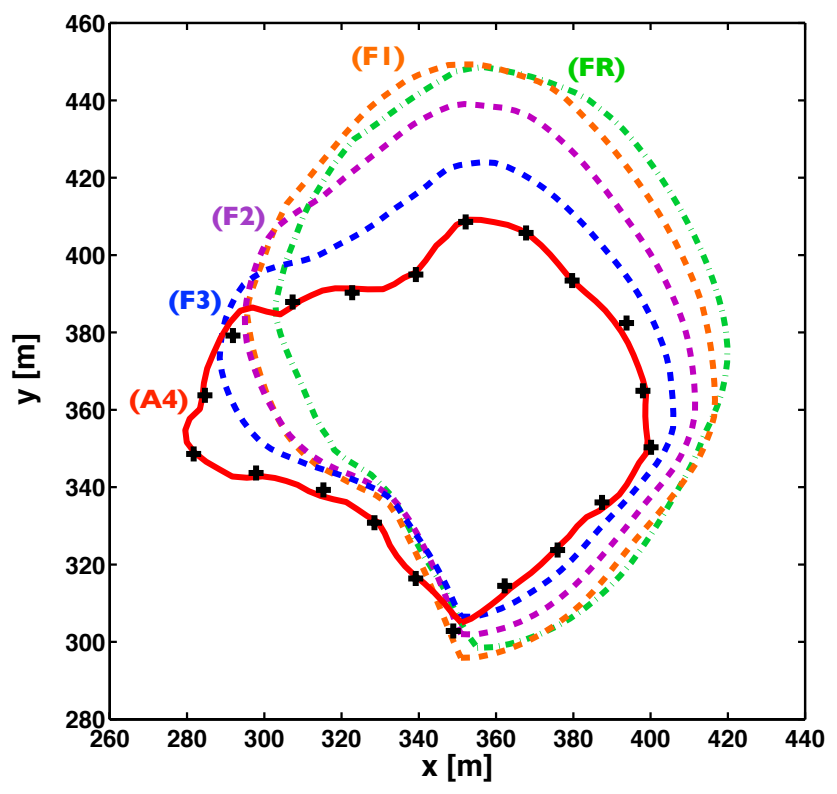

(b)

Figure 12. OSSE anisotropic case over multiple assimilation cycles (from 0 to $600 \mathrm{~s}$ ). (a) Average distance between the true and forecast/analysis fire front location as a function of the assimilation cycle index: green circles correspond to the free run (FR); triangles, crosses, diamonds and stars correspond to a forecast with an EnKF update at $t_{1}=150 \mathrm{~s}(\mathrm{~F} 1), t_{2}=300 \mathrm{~s}(\mathrm{~F} 2), t_{3}=450 \mathrm{~s}(\mathrm{~F} 3)$ and $t_{4}=600 \mathrm{~s}(\mathrm{~F} 4)$, respectively; square symbols correspond to an EnKF update performed at times $t_{1}$ (A1), $t_{2}$ (A2), $t_{3}$ (A3), $t_{4}$ (A4) and $t_{5}$ (A5). (b) Comparison at time $t_{4}=600 \mathrm{~s}$ between the free run $(\mathrm{FR})$ in green dashed-dotted line, the mean forecast estimate (F1, F2, F3) in dashed lines, observations in black crosses, and the mean analysis estimate (A4) in red solid line.
Table 4. Statistical properties of the forecast ensemble in the controlled grassland fire experiment.

\begin{tabular}{lrr}
\hline Random variable & Ensemble mean & Ensemble STD \\
\hline$M_{\mathrm{V}}(\%)$ & 22 & 6 \\
$\Sigma_{\mathrm{V}}\left(\mathrm{m}^{-1}\right)$ & 11500 & 4000 \\
$\left(u_{\mathrm{W}}^{*}, \alpha_{\mathrm{W}}^{*}\right)\left(\mathrm{m} \mathrm{s}^{-1},{ }^{\circ}\right)$ & $(1,307)$ & $(0.4,45)$ \\
$\delta_{\mathrm{V}, 1}(\mathrm{~m})$ & 0.06 & 0.04 \\
$\delta_{\mathrm{V}, 2}(\mathrm{~m})$ & 0.08 & 0.04 \\
$\delta_{\mathrm{v}, 3}(\mathrm{~m})$ & 0.10 & 0.04 \\
$\delta_{\mathrm{V}, 4}(\mathrm{~m})$ & 0.12 & 0.04 \\
$x_{\text {ign }}(\mathrm{m})$ & 2 & 0.65 \\
\hline
\end{tabular}

$600 \mathrm{~K}$ iso-temperature contour) and thereby, provide full fire contours. The study considers four successive, 14 s-long, assimilation cycles with initialization at time $t_{0}=50 \mathrm{~s}$ and successive updates at times $t_{1}=64 \mathrm{~s}, t_{2}=78 \mathrm{~s}, t_{3}=92 \mathrm{~s}$ and $t_{4}=106 \mathrm{~s}$. Based on the spatial resolution of the camera, the estimated STD of the measurement error is $\sigma^{\circ}=0.05 \mathrm{~m}$.

\subsection{Performance of state estimation}

To generate the ensemble of forecasts, the mean values of the ROS model parameters are the (approximately) known short grass properties and wind conditions. Spatially uniform distributions for $M_{\mathrm{v}}, \Sigma_{\mathrm{v}}, u_{\mathrm{w}}^{*}$ and $\alpha_{\mathrm{w}}^{*}$ are assumed; in addition, the grassland lot is divided into four equally sized rectangular-shaped sections and the fuel depth $\delta_{\mathrm{v}}$ is treated as different in each zone, $6,8,10$ and $12 \mathrm{~cm}$ from east to west (noted $\delta_{\mathrm{v}, i}$, with $i=1, \cdots, 4$, respectively). The uncertainty in the initial position of the fire at time $t_{0}$ (along the $x$-direction) is also accounted for; the mean is the observed fire front location taken from the experiment. The ensemble contains $N_{\mathrm{e}}=50$ members corresponding to 9 different choices of the ROS model parameters and of the fire's initial location, i.e., $\left[\delta_{\mathrm{v}, 1}, \delta_{\mathrm{v}, 2}, \delta_{\mathrm{v}, 3}, \delta_{\mathrm{v}, 4}, M_{\mathrm{v}}, \Sigma_{\mathrm{v}}, u_{\mathrm{w}}^{*}, \alpha_{\mathrm{w}}^{*}, x_{\mathrm{ign}}\right]$. The corresponding stochastic perturbations are characterized by relatively large levels as presented in Table 4. In these simulations, $N_{\mathrm{fr}}=100$ markers are used to represent simulated fire fronts and $N_{\mathrm{fr}}^{\mathrm{o}}=50$ markers are considered for observations (i.e., $r=2$ ).

Figure 13a presents a comparison between the mean (ensemble-average) forecast estimate of the fire front location (as predicted by FIREFLY starting from time $t_{0}=50 \mathrm{~s}$ ), the observations and the mean analysis estimate at time $t_{1}=$ $64 \mathrm{~s}$. It is found that the mean free forecast (without DA) significantly underestimates the observed ROS of the fire. In contrast, the predictions that are made after an EnKF update performed at time $t_{1}$ successfully reduce the distance between FIREFLY simulations and observations. In particular, the mean analysis estimate features a topology that is very consistent with the observed fire front, a result that requires an accurate and non-uniform correction of the fire front location. Figure 14 presents the univariate error correlations asso- 

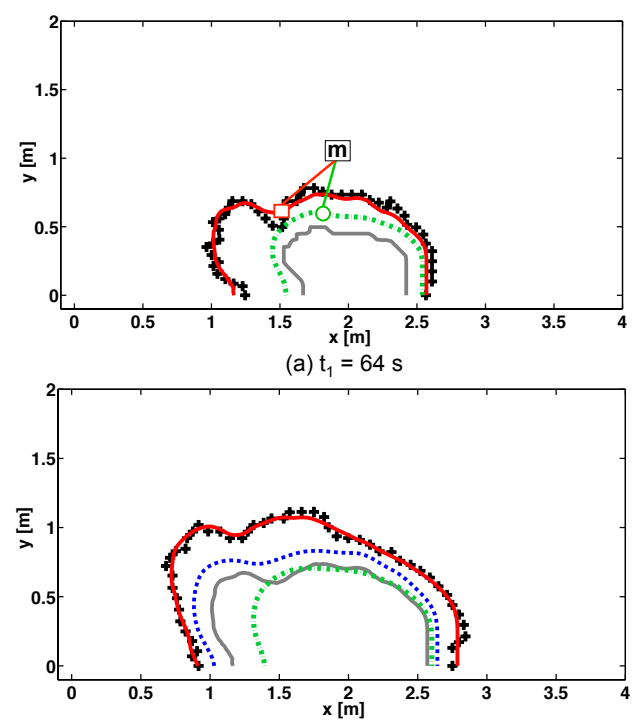

(b) $\mathrm{t}_{2}=78 \mathrm{~s}$

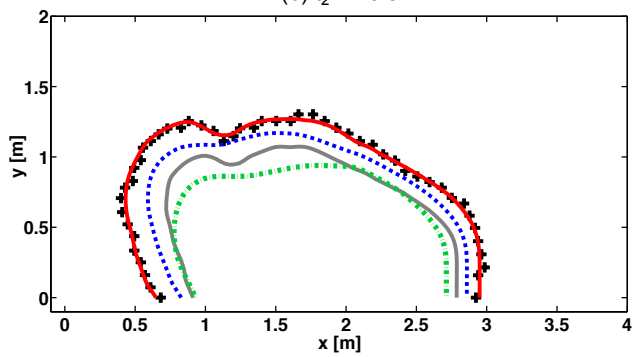

(c) $\mathrm{t}_{3}=92 \mathrm{~s}$

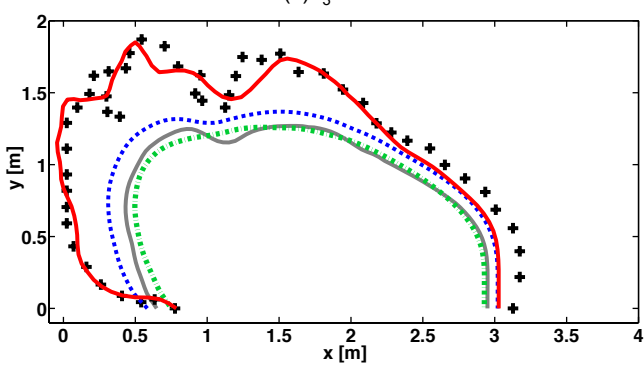

(d) $\mathrm{t}_{4}=106 \mathrm{~s}$

Figure 13. Controlled grassland fire experiment with multiple assimilation cycles from $t_{0}=50 \mathrm{~s}$ to $t_{4}=106 \mathrm{~s}$. Black crosses correspond to observations, the gray solid line corresponds to the initial condition of the assimilation cycle, the green dashed-dotted line corresponds to the free run (without DA), the blue dashed line corresponds to the mean forecast estimate (without DA for the first observation time or with DA at the previous observation time), and the red solid line corresponds to the mean analysis estimate (with a DA update at the current observation time). Assimilation cycles (a) $[50 ; 64 \mathrm{~s}]$; (b) $[64 ; 78 \mathrm{~s}]$; (c) $[78 ; 92 \mathrm{~s}]$; and (d) $[92 ; 106 \mathrm{~s}]$.

ciated with the front marker indexed by $m$ in Fig. 13a for the forecast (blue dashed line) and analysis (red solid line) estimates. The analysis estimates feature a much reduced length scale compared to the forecast estimates to allow for a spatially distributed correction during the EnKF update.

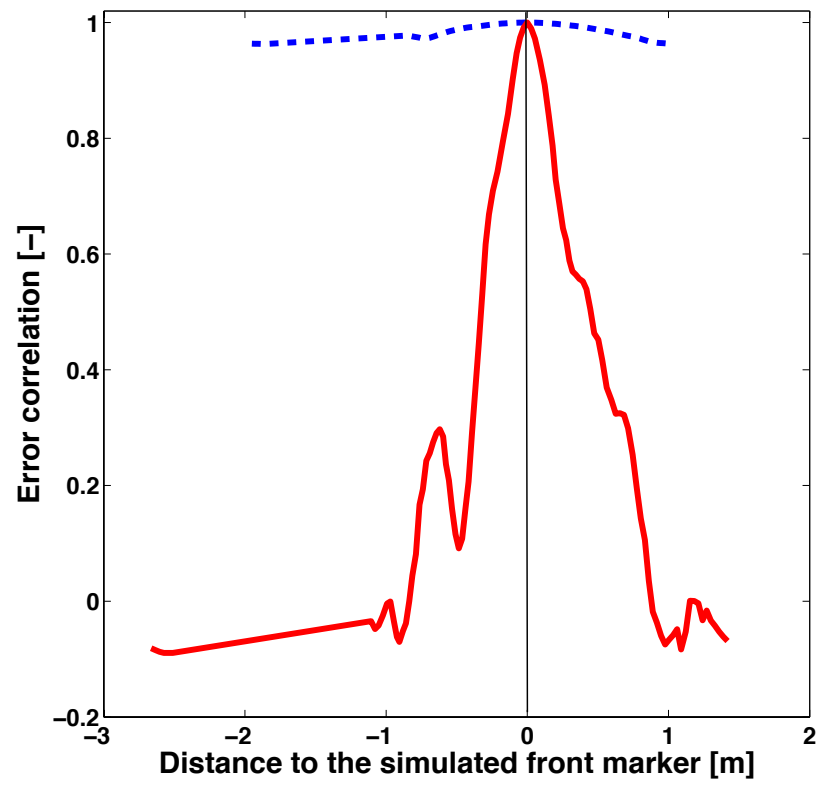

(a)

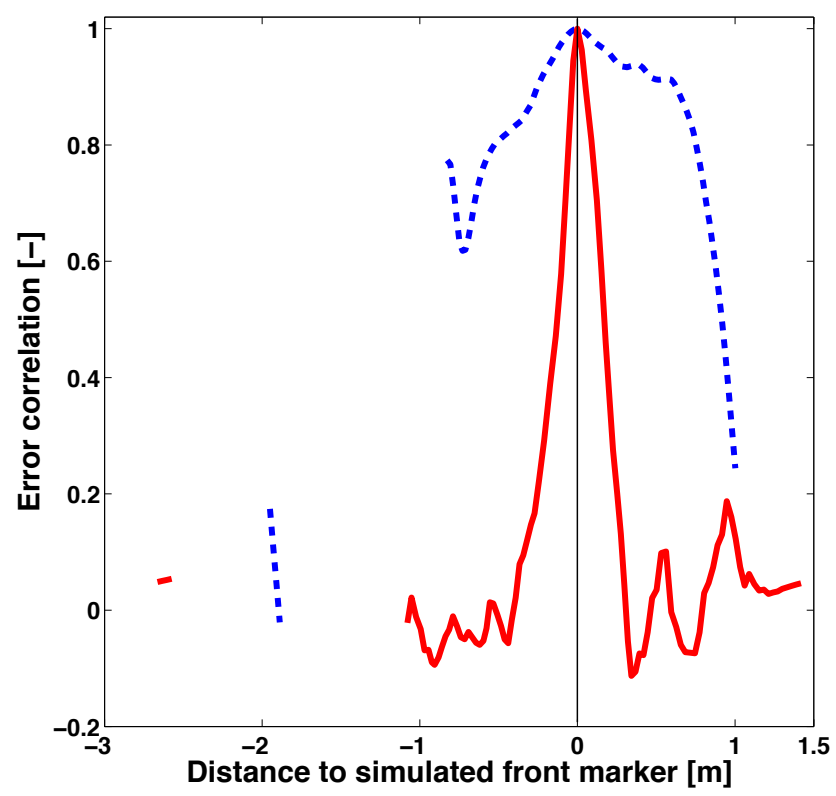

(b)

Figure 14. Univariate error correlation functions along the fireline associated to one marker location (the vertical bar represents its reference location indexed by $\mathrm{m}$ in Fig. 13a) - controlled grassland fire experiment over the assimilation cycle [50; $64 \mathrm{~s}$ ]. (a) Error correlation of the reference marker $x$ coordinate with respect to the errors in the $x$ coordinates of the other markers along the fireline: the blue dashed (red solid) line corresponds to the mean forecast (analysis) estimate. (b) Error correlation of the reference marker $y$ coordinate with respect to the errors in the $y$ coordinates of the other markers along the fireline: the blue dashed (red solid) line corresponds to the mean forecast (analysis) estimate. 
Similar comparisons to Fig. 13a are presented in Fig. 13b$\mathrm{d}$ at times $t_{2}, t_{3}$ and $t_{4}$, respectively. Note that the mean forecast estimate (blue dashed line) is initialized at the previous observation time by the analysis produced by the DA cycle, while the free forecast (green dashed-dotted line) is initialized at time $t_{0}$ and does not use any analysis. It is found that the mean distance to the observations is reduced by a factor of at least 5 over all assimilation cycles, by performing EnKF updates at $14 \mathrm{~s}$ time intervals. Thus, the agreement between predicted and observed front positions is remarkable and significantly better than the level of agreement that would be obtained in the absence of DA. However, in spite of the quality of the correction provided by the SE-based EnKF algorithm, the performance of the forecast remains limited; for instance, Fig. $13 \mathrm{~b}$ shows that the mean forecast estimate (initialized by the analysis at time $t_{1}$ and integrated until time $t_{2}$ ), while still significantly more accurate than the free forecast (initialized by the initial conditions at time $t_{0}$ and integrated until time $t_{2}$ ), is not in good agreement with the observation (relatively to the mean analysis estimate). These results suggest that in the present configuration, the persistence of the initial condition is limited to short lead-times (i.e., shorter than $14 \mathrm{~s}$ ) and the performance of the SE-based EnKF forecast could be improved by more frequent assimilation.

\subsection{Comparison to parameter estimation}

A PE approach based on the standard EnKF approach and validated in Rochoux et al. (2014a) is applied to the same controlled grassland fire experiment. While the SE approach leads to a direct adjustment of the fire front location, the PE approach works by an adjustment of the ROS model parameters. In the PE approach, four parameters are used as control variables: the fuel moisture content $M_{\mathrm{V}}$ and particle surfaceto-volume ratio $\Sigma_{\mathrm{v}}$ as well as the wind magnitude $u_{\mathrm{w}}^{*}$ and direction angle $\alpha_{\mathrm{w}}^{*}$, i.e.,

$\boldsymbol{x}=\left[M_{\mathrm{v}}, \Sigma_{\mathrm{v}}, u_{\mathrm{w}}^{*}, \alpha_{\mathrm{w}}^{*}\right]$,

with $n=4$ the size of the control vector. These parameters are perturbed around mean values and with prescribed uncertainties (according to assumed levels of uncertainty presented in Table 4), but remain spatially uniform for each ensemble member. The EnKF ensemble contains $N_{\mathrm{e}}=1000$ members, meaning that during each assimilation cycle, FIREFLY produces 1000 fire front trajectories associated with each set of control parameters. Note that the size of the ensemble is drastically increased compared to the SE approach, in order to retrieve accurate error statistics of the control parameters and to avoid the equifinality problem (i.e., a problem in which multiple sets of parameters provide the same simulated fire front), see Rochoux (2014) for further explanations on this equifinality problem.

Figure 15a compares the mean forecast estimate obtained at time $t_{4}=106 \mathrm{~s}$, using the PE ensemble (blue dashed line) and the SE ensemble (blue solid line) as predicted by FIRE-
FLY integration and with an EnKF update performed at successive observation times $t_{1}=64 \mathrm{~s}, t_{2}=78 \mathrm{~s}$ and $t_{3}=92 \mathrm{~s}$. Figure $15 \mathrm{~b}$ compares the mean analysis estimate obtained at the same time $t_{4}=106 \mathrm{~s}$ using a PE-based EnKF update (red dashed line) and a SE-based EnKF update (red solid line). In both figures, predictions are compared to observations (black crosses). It is found that for both PE and SE approaches, the analysis estimates provide more accurate fire front locations than the forecast estimates. This point is also illustrated in Fig. 16, which presents the RMS distance between the observations and the mean fire front location produced by the forecast estimates (see Fig. 16a) and analysis estimates (see Fig. 16b) over the four successive assimilation cycles; the mean distance to the observations is reduced by a factor 2 in the PE approach and by a factor of at least 5 in the SE approach. Additionally, the performance of the SE-based analysis is significantly better than that of the PE-based analysis (relying on spatially uniform distributions of ROS model parameters); the mean distance between observed and SEbased simulated fire fronts remains below $0.1 \mathrm{~m}$ for all assimilation cycles and the scatter of the SE-based analysis ensemble is significantly less than that obtained through PE.

In spite of the overall quality of the correction provided by both EnKF estimation approaches, the accuracy of the forecast, while still significantly better than that obtained in the free run simulations, rapidly decreases over time. Some of the benefits of an analysis are indeed lost in the forecast at time $t_{4}$, due to the limited persistence of the initial condition and/or due to the temporal variability of the errors in the environmental conditions. The improved forecast performance of the PE approach is illustrated in Fig. 16a; the adjustment of the ROS model parameters allows for a correction of inaccuracies in initial guesses as well as an adaptation to timedependent properties; the statistical properties of the EnKF ensemble are dynamically evolving. In contrast, the statistical properties of the SE-based EnKF ensemble are constant. Figure 17 illustrates this point. In particular, this figure shows that the prior values of the moisture content $M_{\mathrm{v}}$ and the fuel particle surface-to-volume ratio $\Sigma_{\mathrm{v}}$ are not adequate to effectively track the actual fire propagation (in comparison, the estimated wind conditions in the PE approach are contained in the support of the forecast PDFs). These values are corrected in the PE approach; the mean (ensemble-average) value of $M_{\mathrm{v}}$ is decreased from $22 \%$ to approximately $10 \%$; the mean (ensemble-average) value of $\Sigma_{\mathrm{v}}$ is increased from $11500 \mathrm{~m}^{-1}$ to approximately $19000 \mathrm{~m}^{-1}$. These values are not corrected in the SE approach and therefore, induce a significant bias in wildfire spread simulations.

In this validation study, the assimilation needs to be renewed by frequent observations with an assimilation frequency (i.e., frequency at which the EnKF update is renewed by observations) that is high enough to track the temporal variability of the errors in the ROS model parameters. Updating environmental conditions (the biomass moisture content 


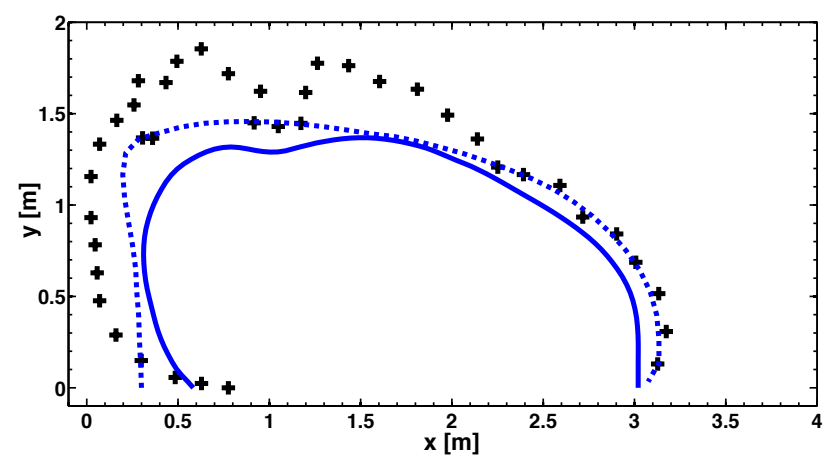

(a) Forecast over [92; $106 \mathrm{~s}]$

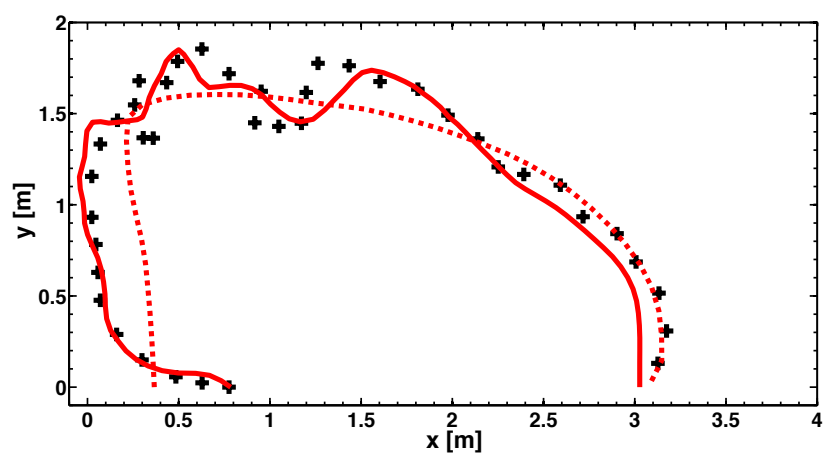

(b) Analysis at $\mathrm{t}_{4}=106 \mathrm{~s}$

Figure 15. Comparison between simulated (lines) and observed (symbols) front positions at time $t_{4}=106 \mathrm{~s}$ in the controlled grassland burning experiment. The simulated front position is the mean position calculated as the average of the EnKF ensemble; dashed lines (solid lines) correspond to the PE-based (SE-based) simulations. (a) Forecast (with EnKF update at $t_{3}=92 \mathrm{~s}$ ). (b) Analysis (with EnKF update at $t_{4}=106 \mathrm{~s}$ ).

in particular) explains the bulk of the improved forecasts at future lead times.

\section{Conclusions}

This study is the second part in a series of two articles, in which we propose and explore a new paradigm for producing optimized forecasts of the wildfire behavior based on observations of the fire front location. The prototype data-driven simulator combines a regional-scale wildfire spread model, FIREFLY, with a data assimilation (DA) algorithm based on an ensemble Kalman filter (EnKF); it features a choice between a parameter estimation (PE) approach (in which the estimation targets are the parameters of the rate of spread (ROS) model) and a state estimation (SE) approach (in which the estimation targets are the time-evolving spatial coordinates of the fire front). The cornerstone of this prototype data-driven simulator is to do the following:

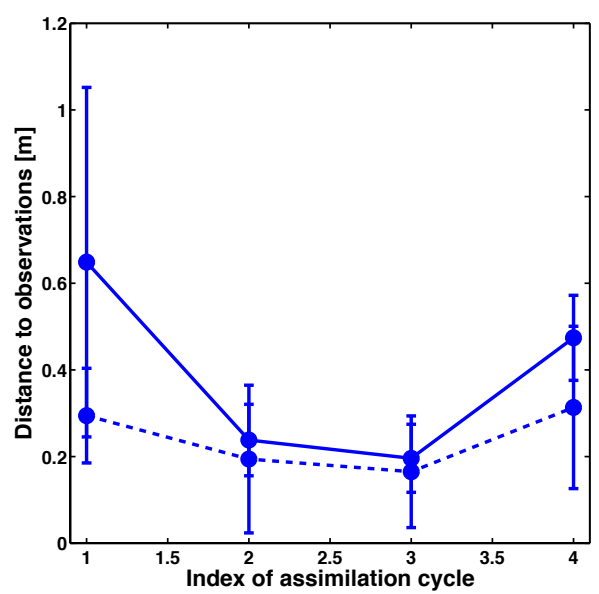

(a) Forecast RMS distance to observations

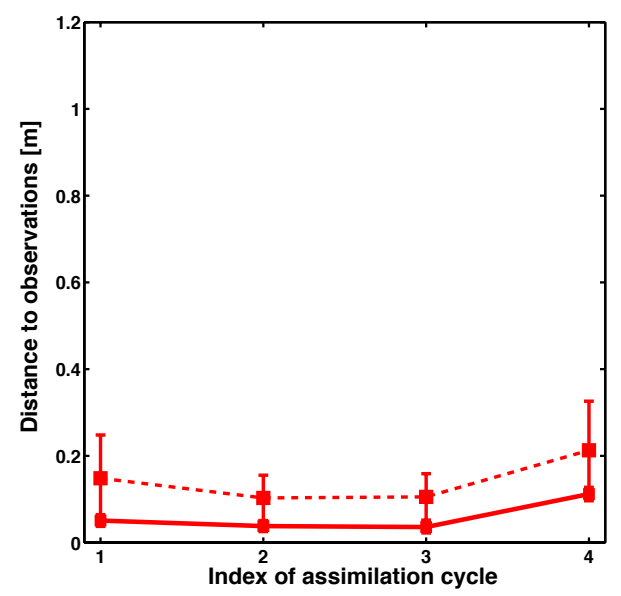

(b) Analysis RMS distance to observations

Figure 16. Evolution over 4 successive assimilation cycles of the average distance between the observations and the mean fire front location produced by the (a) forecast estimates and the (b) analysis estimates at times $t_{1}=64 \mathrm{~s}, t_{2}=78 \mathrm{~s}, t_{3}=92 \mathrm{~s}$, and $t_{4}=106 \mathrm{~s}$ Dashed (solid) lines represent PE (SE) results; vertical bars indicate the STD in the forecast and analysis estimates.

1. explicitly account for the effects of both measurement and modeling errors and thereby, overcome some of the current limitations of regional-scale wildfire modeling;

2. account for non-linearities in the wildfire behavior and for temporal variability of the errors in the environmental conditions;

3. forecast reliable wildfire spread scenarios at limited computational cost, consistently with an operational framework for real-time monitoring of wildfire behavior.

The present study assumes that airborne and/or spaceborne observations of the fire front location are available at frequent times but possibly provide an inaccurate and incomplete description of the fire front due to the opacity of the 


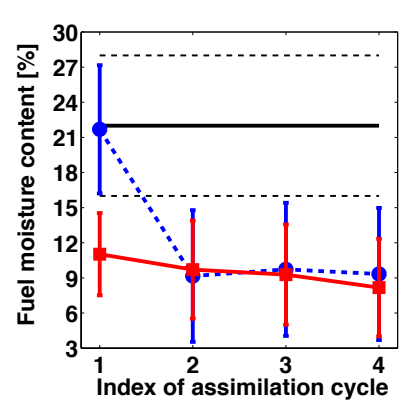

(a)

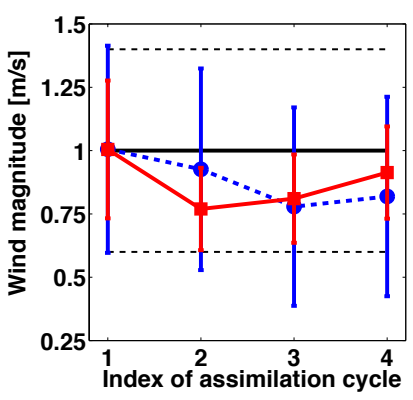

(c)

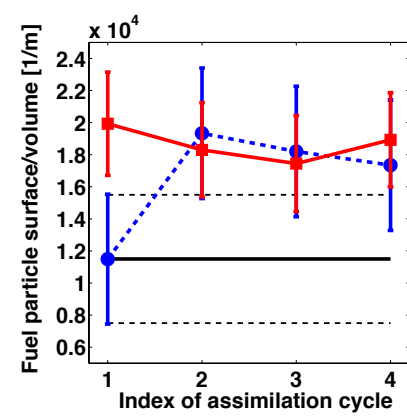

(b)

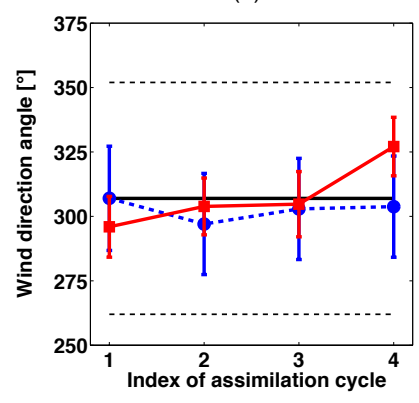

(d)
Figure 17. Evolution of ROS model parameters at four successive analysis times; controlled grassland fire experiment. PE-based ensemble: blue circle symbols (connected by a dashed line) indicate mean values in the forecast ensemble; red square symbols (connected by a solid line) indicate mean values in the analysis ensemble; vertical bars indicate the corresponding STD. SE-based ensemble: black horizontal solid lines indicate mean values; black horizontal dashed lines indicate STD. (a) Biomass fuel moisture content $M_{\mathrm{V}}(\%)$. (b) Biomass fuel particle surface-to-volume ratio $\Sigma_{\mathrm{V}}\left(\mathrm{m}^{-1}\right)$. (c) Wind magnitude $u_{\mathrm{W}}^{*}\left(\mathrm{~m} \mathrm{~s}^{-1}\right)$. (d) Wind direction $\alpha_{\mathrm{W}}^{*}\left({ }^{\circ}\right)$.

fire-induced thermal plume or due to a limited monitoring. The performance of both PE and SE approaches was evaluated on synthetic cases of wildfire spread that are representative of real wildfire conditions as well as on a reduced-scale controlled grassland fire experiment.

Even though certain input parameters of the ROS model can be assumed constant over the fire duration (in particular, intrinsic properties of biomass fuels), other parameters exhibit a dynamic behavior due to the presence of the propagating wildfire. Wind conditions change over time, partly due to the fire/atmosphere interactions and their feedback on local atmospheric conditions (in terms of wind, air temperature and humidity). In addition, the moisture content of biomass fuel also varies, in particular that of dead biomass, which can be considered in thermal equilibrium with the atmosphere. A dynamic estimation of time-varying environmental parameters is therefore required to produce accurate simulations of the wildfire behavior. For this purpose, a PE approach was presented in Part I of this series of two articles, see Rochoux et al. (2014a). This PE approach was restricted to spatially uniform parameters. Accounting for the detailed spatial variations of environmental conditions would indeed significantly increase the computational cost, with no means of assessing the consistency of the EnKF update to in situ measurements (since those are usually provided with a coarse spatial resolution).

Part II of this series of two articles demonstrated that to obtain a local correction of the fire front location, the generation of the stochastic ensemble must represent the anisotropy in wildfire spread. This anisotropy was implicitly introduced in the SE approach by selecting spatially distributed biomass fuel properties and distinct wind conditions between the different members in the ensemble. The SE approach was shown to be successful at retrieving at low computational cost (i.e., with a relatively small number of members in the ensemble), the actual shape of the fire front in cases with strong anisotropic propagation conditions. This approach was also found relevant for observations made with significant errors and/or cases in which the observations are incomplete (e.g., when only a section of the fireline is informed), in order to reconstruct a complete, reliable initial condition for FIREFLY restart.

Furthermore, it was demonstrated that for the present controlled grassland fire experiment, the PE approach provides a more reliable forecast capability of the wildfire behavior than the SE approach (due to improved knowledge in environmental conditions that are usually poorly assessed) at short lead times (i.e., at $14 \mathrm{~s}$ time intervals). This ranking between PE and SE approaches remains problem-dependent and may not hold in cases where the assimilation cycle is longer (due to a lower observation frequency) and where the values of the environmental conditions strongly fluctuate during an assimilation cycle. The duration of the assimilation cycle (i.e., a user-defined variable) is therefore of primary importance in the success of the proposed DA approaches; it must be specified consistently with the persistence of the initial condition and/or with the temporal and spatial variability of the errors in the environmental conditions.

This series of two articles emphasizes the potential of DA to dramatically increase wildfire simulation accuracy. In particular, the present original comparison between different data assimilation strategies show that the future data-driven wildfire spread strategy will be a blend of the PE and SE strategies in order to address all possible sources of uncertainties in the fire representation, consistently with the available amount of information on the fire event.

While wildfire spread forecast capabilities are still at an early stage of development, it is envisioned that they will be similar to current weather forecast capabilities and that they will provide real-time fire forecasts using thermal-infrared imaging including a description of both wildfire dynamics and plume emissions such as FOREFIRE-MESONH (Filippi et al., 2009, 2013). Thus, future plans include the following:

- the extension of the FIREFLY front-tracking simulator to complex terrain topography; a recent study (Ro- 
choux et al., 2014b) has shown that the performance of the EnKF algorithm is preserved for synthetic cases of slope-aided wildfire spread and that the DA formalism remains the same as for a no-slope fire propagation; but this needs to be validated against real-world wildfire events;

- the evaluation of the PE/SE-based estimation strategy for real-world wildfire events, in order to design the observation requirements (in terms of spatial and temporal resolutions) to obtain reliable forecasts of wildfire spread;

- the development of a dual SE/PE approach that could partly overcome the forecast limitations illustrated in the present and past studies (the PE approach could be extended to the case of weak spatial variations of the ROS model parameters);

- the study of the forecast performance with respect to the formulation of the ROS model and the possible generation of the ensemble with different ROS models (the Rothermel's semi-empirical model was used in the present study to demonstrate the feasibility of the EnKF algorithm, but the present strategy could be applied to any ROS model in the literature);

- the integration of the DA algorithm into a meso-scale atmospheric model based on computational fluid dynamics. The issues related to DA for coupled physical systems were not addressed in this work. We propose here a DA strategy that is suitable for surface wildfire spread, but that would need further developments to deal with a land surface-atmosphere coupled modeling system.

In summary, this preliminary investigation demonstrates the feasibility of data assimilation for wildfire spread forecasting, and provides useful guidelines to design the future capabilities of the data-driven prediction system. The complexity of the system will be gradually increased and validated against more realistic wildfire events, in order to meet the operational requirements of wildfire monitoring in the long term.

Acknowledgements. The financial support provided by the Agence Nationale de la Recherche under the IDEA ${ }^{2}$ project grant ANR-09-COSI-006 (2010-2013) and by the LEFE-MANU grant (INSU-CNRS program, 2011-2013) was greatly appreciated. The authors also gratefully acknowledge Ronan Paugam and Martin Wooster (King's College London) for sharing the experimental data, Florent Duchaine and Thierry Morel (CERFACS) for support on OpenPALM, Albert Simeoni (University of Edinburgh) for clarifications on Rothermel's model and helpful discussions on wildfire spread modeling, as well as Jonathan Beezley for helpful discussions on state estimation problems for wildfire spread.

\footnotetext{
${ }^{2}$ http://anridea.univ-corse.fr
}

Edited by: R. Lasaponara

\section{References}

Andrews, P. L., Cruz, M. G., and Rothermel, R. C.: Examination of the wind speed limit function in the Rothermel surface fire spread model, Int. J. Wildland Fire, 22, 959-969, 2013.

Balbi, J.-H., Morandini, F., Silvani, X., Filippi, J.-B., and Rinieri, F.: A physical model for wildland fires, Comb. Flame, 156, 2217 2230, 2009.

Buis, S., Piacentini, A., and Declat, D.: PALM: a computational framework for assembling high performance computing applications, Concurr. Comp.-Pract. E., 18, 247-262, 2006.

Burgan, R.: 1988 revisions to the 1978 national fire-danger rating system, Technical Report Research Paper SE-273, US Department of Agriculture, Forest Service, Southeastern Forest Experiment Station, Asheville, NC, USA, 1988.

Burgers, G., van Leeuwen, P., and Evensen, G.: Analysis scheme in the ensemble kalman filter, Mon. Weather Rev., 126, 1719-1724, 1998.

Chandler, C., Cheney, N., Thomas, P., Trabaud, L., and Williams, D.: Fire in Forestry. Volume I: Forest Fire Behavior and Effects, John Wiley \& Sons, New York, 1983.

Chong, D., Tolhurst, K. G., Duff, T. J., and Cirulis, B.: Sensitivity analysis of PHOENIX RapidFire, Bushfire CRC, University of Melbourne, 2013.

Daley, R.: Atmospheric Data Analysis, Cambridge University Press, Cambridge, 1991.

Dowdy, A., Mills, G., Finkele, K., and de Groot, W.: Australian fire weather as represented by the McArthur forest fire danger index and the Canadian forest fire weather index, Technical Report CAWCR Technical Report No. 10, Centre for Australian Weather and Climate Research, 2009.

Filippi, J.-B., Bosseur, F., Mari, C., Lac, C., Le Moigne, P., Cuenot, B., Veynante, D., Cariolle, D., and Balbi, J.-H.: Coupled atmosphere-wildland fire modelling, J. Adv. Model. Earth Syst., 1, 210-226, 2009.

Filippi, J.-B., Pialat, X., and Clements, C.: Assessment of FOREFIRE/MESONH for wildland fire/atmosphere coupled simulation of the FireFlux experiment, Proc. Combust. Inst., 34, 2633 2640, 2013.

Finney, M. A.: FARSITE: Fire area simulator - model development and evaluation, Forest Service, US Dept. of Agriculture, Research Paper RMRS-RP-4, 1998.

Finney, M. A., Grenfell, I. C., McHugh, C. W., Seli, R. C., Trethewey, D., Stratton, R. D., and Brittain, S.: A method for ensemble wildland fire simulation, Environ. Model. Assess., 16, 153-167, 2011.

Fouilloux, A. and Piacentini, A.: The PALM project: MPMD paradigm for an oceanic data assimilation software, Lect. Notes Comput. Sc., 1685, 1423-1430, 1999.

Hirsch, K.: Canadian forest fire behavior prediction (FBP) system: User's guide, Technical report, Northern Forest Centre, Special Report No. 7, 1996.

Kochanski, A. K., Jenkins, M. A., Mandel, J., Beezley, J. D., and Krueger, S. K.: Real time simulation of 2007 Santa Ana fires, Forest Ecol. Manag., 294, 136-149, 2013. 
Lagarde, T., Piacentini, A., and Thual, O.: A new representation of data assimilation methods: the PALM flow charting approach, Q. J. Roy. Meteor. Soc., 127, 189-207, 2001.

Linn, R., Reisner, J., Colman, J. J., and Winterkamp, J.: Studying wildfire behavior using FIRETEC, Int. J. Wildland Fire, 11, 233246, 2002.

Mandel, J. and Beezley, J. D.: Morphing Ensemble Kalman filter, Tellus A, 60, 131-140, doi:10.1111/j.1600-0870.2007.00275.x, 2007.

Mandel, J., Bennethum, L. S., Beezley, J. D., Coen, J. L., Douglas, C. C., Minjeong, K., and Vodacek, A.: A wildland fire model with data assimilation, Math. Comput. Simulat., 79, 584606, 2008.

Mandel, J., Beezley, J. D., and Kochanski, A. K.: Coupled atmosphere-wildland fire modeling with WRF 3.3 and SFIRE 2011, Geosci. Model Dev., 4, 591-610, doi:10.5194/gmd-4-5912011, 2011.

Mell, W., Jenkins, M. A., Gould, J., and Cheney, P.: A physics-based approach to modeling grassland fires, Int. J. Wildland Fire, 16, $1-22,2007$.

Noble, I., Bary, G., and Gill, A.: McArthur's fire danger meters expressed as equations, Aust. J. Ecol., 5, 201-203, 1980.

Pannekoucke, O., Berre, L., and Desroziers, G.: Background error correlation length-scale estimates and their sampling statistics, Q. J. Roy. Meteor. Soc., 134, 497-511, 2008.

Paugam, R., Wooster, M. J., and Roberts, G.: Use of handheld thermal imager data for airborne mapping of fire radiative power and energy and flame front rate of spread, IEEE T. Geosci. Remote, 51, 3385-3399, 2013.

Riggan, P. and Robert, G.: Airborne remote sensing of wildland fires, in: Wildland Fires and Air Pollution, edited by: Bytnerowicz, A., Arbaugh, M., Andersen, C., and Riebau, A., Developments in Environmental Science 8, Elsevier, 139-168, 2009.

Rochoux, M. C.: Vers une meilleure prévision de la propagation d'incendies de forêt: evaluation de modèles et assimilation de données, Ph.D. Thesis (written in English: Towards a more comprehensive monitoring of wildfire spread - Contributions of model evaluation and data assimilation strategies), Ecole Centrale Paris (France), 2014.

Rochoux, M. C., Ricci, S., Lucor, D., Cuenot, B., Trouvé, A., and Bart, J.-M.: Towards predictive simulations of wildfire spread using a reduced-cost Ensemble Kalman filter based on Polynomial Chaos approximations, Proceedings of the Summer Program, Center for Turbulence Research, July 2012, NASA AMES, Stanford University, USA, 2012.

Rochoux, M. C., Cuenot, B., Ricci, S., Trouvé, A., Delmotte, B., Massart, S., Paoli, R., and Paugam, R.: Data assimilation applied to combustion, C. R. Mecanique, 341, 266-276, 2013a.
Rochoux, M. C., Delmotte, B., Cuenot, B., Ricci, S., and Trouvé, A.: Regional-scale simulations of wildland fire spread informed by real-time flame front observations, Proc. Combust. Inst., 34, 2641-2647, $2013 b$.

Rochoux, M. C., Ricci, S., Lucor, D., Cuenot, B., and Trouvé, A.: Towards predictive data-driven simulations of wildfire spread - Part I: Reduced-cost ensemble Kalman filter based on polynomial chaos surrogate model for parameter estimation, Nat. Hazards Earth Syst. Sci., 14, 2951-2973, doi:10.5194/nhess-142951-2014, 2014a.

Rochoux, M. C., Emery, C., Ricci, S., Cuenot, B., and Trouvé, A.: Comparative study of parameter estimation and state estimation approaches in data-driven wildfire spread modeling, VII International Conference on Forest Fire Research, Coimbra (Portugal), 14-20 November, 2014b.

Rothermel, R. C.: A mathematical model for predicting fire spread in wildland fuels, USDA Forest Service, Research Paper INT-115, Intermountain Forest and Range Experiment, Ogden, UT:40, 1972.

Sandberg, D .V., Riccardi, C. L., and Schaaf, M. D.: Reformulation of Rothermel's wildland fire behaviour model for heterogeneous fuelbeds, Can. J. Forest Res., 37, 2438-2455, doi:10.1139/X07094, 2007.

Sullivan, A. L.: Wildland surface fire spread modeling, 1990-2007: 2. Empirical and quasi-empirical models, Int. J. Wildland Fire, 18, 369-386, 2009.

Tymstra, C., Bryce, R. W., Wotton, B. M., Taylor, S. W., and Armitage, O. B.: Development and structure of Prometheus: the Canadian Wildland Fire Growth Simulation Model, Natural Resources Canada, Canadian Forest Service, Northern Forestry Centre, Edmonton, Alberta, Information Report NOR-X-417, 2010.

Van Wagner, C.: Development and structure of the canadian forest fire weather index system, Technical Report 35, Canadian Forestry Service, Ottawa, ON, Canada, 1987.

Wooster, M. J., Roberts, G., Perry, G., and Kaufman, Y. J.: Retrieval of biomass combustion rates and totals from fire radiative power observations: FRP derivation and calibration relationships between biomass consumption and fire radiative energy release, J. Geophys. Res., 110, D24311, doi:10.1029/2005JD006318, 2005.

Wooster, M., Robert, G., Smith, A., Johnston, J., Freeborn, P., Amici, S., and Hudak, A.: Thermal infrared remote sensing, in: Thermal Remote Sensing of Active Vegetation Fires and Biomass Burning Events, Volume Remote Sensing and Digital Image Processing 17, Springer, 347-390, 2013. 